\title{
Pathogen Recognition and Activation of the Innate Immune Response in Zebrafish
}

\author{
Michiel van der Vaart, Herman P. Spaink, and Annemarie H. Meijer \\ Institute of Biology, Leiden University, Einsteinweg 55, 2333 CC Leiden, The Netherlands \\ Correspondence should be addressed to Annemarie H. Meijer, a.h.meijer@biology.leidenuniv.nl
}

Received 3 February 2012; Accepted 22 April 2012

Academic Editor: Christopher Hall

Copyright ( $) 2012$ Michiel van der Vaart et al. This is an open access article distributed under the Creative Commons Attribution License, which permits unrestricted use, distribution, and reproduction in any medium, provided the original work is properly cited.

\begin{abstract}
The zebrafish has proven itself as an excellent model to study vertebrate innate immunity. It presents us with possibilities for in vivo imaging of host-pathogen interactions which are unparalleled in mammalian model systems. In addition, its suitability for genetic approaches is providing new insights on the mechanisms underlying the innate immune response. Here, we review the pattern recognition receptors that identify invading microbes, as well as the innate immune effector mechanisms that they activate in zebrafish embryos. We compare the current knowledge about these processes in mammalian models and zebrafish and discuss recent studies using zebrafish infection models that have advanced our general understanding of the innate immune system. Furthermore, we use transcriptome analysis of zebrafish infected with E. tarda, S. typhimurium, and M. marinum to visualize the gene expression profiles resulting from these infections. Our data illustrate that the two acute disease-causing pathogens, $E$. tarda and S. typhimurium, elicit a highly similar proinflammatory gene induction profile, while the chronic disease-causing pathogen, M. marinum, induces a weaker and delayed innate immune response.
\end{abstract}

\section{Introduction}

The use of adult zebrafish (Danio rerio) and their transparent offspring as hosts to model infectious diseases caused by human pathogens, or closely related animal pathogens, has recently provided novel insights into pathogenesis, which in many cases could not have been achieved using mammalian models [1-6]. The power of the zebrafish model lies in its suitability for genetic approaches, high-throughput screening, and live imaging studies. Fluorophore-marked transgenic lines are now available that allow unprecedented visualization of pathogen interactions with macrophages and neutrophils, the major phagocytic innate immune cell types of zebrafish larvae [7-11]. As early as one day after fertilization (dpf), zebrafish embryos display phagocytic activity towards microbial infections [12] and are able to mount an innate immune response with a transcriptional signature that resembles responses in mammalian or cell culture systems [13]. Adaptive immunity becomes active after approximately three weeks of development [14]. Therefore, innate immunity can be studied during the earlier zebrafish embryonic and larval stages in the absence of $\mathrm{T}$ - and $\mathrm{B}$-cell responses. In this paper we focus on signaling pathways involved in pathogen recognition and activation of the innate immune response in zebrafish embryos and larvae. We compare the knowledge of the zebrafish innate immune system with that of human and mammalian models and discuss results from transcriptomic analyses that show clear specificity in responses to different bacterial pathogens, such as Salmonella and Mycobacteria species.

\section{Pattern Recognition Receptors}

The innate immune system is the host's first line of defense against infection; therefore, its main role is to recognize invading pathogens early and trigger an appropriate proinflammatory response [15]. The innate immune system utilizes a limited number of germline-encoded pattern recognition receptors (PRRs) to recognize evolutionary conserved structures on pathogens, named pathogen-associated molecular patterns (PAMPs) [15]. PRRs are also capable of indirectly sensing the presence of pathogens $[16,17]$. 
This occurs when infection, inflammation, or other cellular stresses cause host factors to be present in aberrant locations, or to form abnormal molecular complexes, so called dangerassociated molecular patterns (DAMPs) [17]. PRRs located on the cell surface are scouting the extracellular environment for the presence of microbes. PRRs located on endosomes identify microbes that have entered the phagolysosomal degradation pathway, and cytoplasmic PRRs recognize intracellular cytosolic pathogens or components of internalized microbes [18]. Upon PAMP recognition, PRRs signal the presence of infection and initiate proinflammatory and antimicrobial responses by activating several intracellular signaling pathways [19], ultimately leading to activation of gene expression and synthesis of a broad range of molecules. These include proinflammatory and chemotactic cytokines and antimicrobial peptides [20]. The different families of PRRs present in both humans and zebrafish and their downstream signaling pathways are summarized in Figure 1 and will be discussed below.

2.1. Toll-Like Receptors. The most extensively studied class of PRRs are the Toll-like receptors (TLRs), a family of 10 proteins in human. TLRs are named after the Drosophila Toll protein, which functions in dorsoventral patterning and antifungal responses [23]. TLRs are integral glycoproteins which possess an extracellular or luminal, ligand-binding domain with leucine-rich repeat (LRR) motifs and a cytoplasmic signaling Toll/Interleukin-1 (IL-1) receptor homology (TIR) domain $[20,24]$. In mammals, the main cell types expressing TLRs are antigen-presenting cells (APCs), including macrophages and dendritic cells, and B lymphocytes [18]. However, most cell types are capable of expressing TLRs, for instance, in response to a localized infection [25]. In mammals, TLR4 recognizes Gram-negative bacteria via the lipid A portion of lipopolysaccharide (LPS), while TLR2 recognizes Gram-positive bacteria via lipoteichoic acid (LTA), lipoproteins, and peptidoglycan, and TLR5 recognizes the motility apparatus protein flagellin, which can be present on both Gram types [18]. Other TLRs are specialized in recognizing nuclear acids in endosomal and phagosomal compartments. TLR3 can detect viral replication by binding to double-stranded RNA (dsRNA), TLR7 and TLR8 specifically recognize single-stranded RNA (ssRNA) of RNA viruses, and unmethylated CpG DNA present in the genomes of viruses and bacteria is detected by TLR9 [18]. Ligand binding by a TLR will induce it to form homomeric or heteromeric oligomers, which triggers intracellular signal transduction via their TIR domains [18]. The mammalian TLR signaling pathway uses five different TIR-domain-containing adaptor molecules: MYD88, MAL/TIRAP, TRIF/TICAM1, TRAM/TICAM2, and SARM $[19,24]$. Among these, MYD88 is the most universal adaptor, since it is used for downstream signaling by all TLRs, with the exception of TLR3 [26]. Downstream signaling via central intermediate molecules such as TRAF6 will eventually lead to the activation of transcription factors, mostly members of the ATF, NF $\kappa \mathrm{B}$, AP-1, IRF, and STAT families, regulating the expression of a battery of antimicrobial and proinflammatory genes [26].
Putative orthologs of mammalian TLRs have been identified in zebrafish, in addition to some fish-specific family members [27, 28]. A genome duplication during the evolution of teleost fish most likely explains why zebrafish have two counterparts for some of the mammalian TLRs (e.g., tlr $4 b a / t l r 4 b b$ for TLR4 and tlr5a/tlr5b for TLR5), but it is still unknown whether this increase in the number of receptors is associated with diversification in PAMP recognition [4]. Only some of the zebrafish TLR ligands are currently known [29]. The specificity of TLR2, TLR3, and TLR5 is conserved between mammals and fish, recognizing lipopeptides, dsRNA, and flagellin, respectively [13, 30, 31]. Additionally, the fish-specific TLR22 has been shown to recognize dsRNA and PolyI:C [31]. However, zebrafish TLR4 cannot be stimulated by LPS, illustrating that not all ligand specificities are conserved between mammals and zebrafish $[32,33]$. Signaling intermediates in the pathway downstream of mammalian TLRs have also been identified in zebrafish, including homologs of four of the adaptor proteins, Myd88, Mal/Tirap, Trif/Ticam1, and Sarm, and the central intermediate Traf6 [34]. Among these, Myd88 and Traf6 have been functionally studied by knockdown analysis in zebrafish embryos, showing their requirement for a proinflammatory innate immune response to microbial presence [13, 35-37]. Furthermore, triggering of the innate immune response in zebrafish embryos also leads to induction of members of the ATF, NF $\kappa$ B , AP-1, IRF, and STAT families of transcription factors $[13,38]$.

2.2. NOD-Like Receptors. Pathogens that escape the surveillance of cell surface and endosomal PRRs may end up in the cytosol, where nucleotide-binding-oligomerization-domain(NOD-) like receptors (NLRs) detect their presence by intracellular PAMPs and DAMPs [39]. The NLRs constitute a family of 23 proteins in humans. Their defining features are the presence of a centrally located NOD domain responsible for oligomerization, a C-terminal LRR capable of ligandbinding, and an N-terminal protein-protein interaction domain, such as the caspase recruitment domain (CARD), pyrin (PYD), or baculovirus inhibitor repeat (BIR) domain [40]. Two of the NLRs, NOD1 and NOD2, can sense bacterial presence by directly or indirectly detecting molecules produced during synthesis or breakdown of peptidoglycan [40]. NOD1 recognizes g-D-glutamyl-meso-diaminopimelic acid (iE-DAP), a dipeptide produced mostly by Gram-negative bacteria, whilst NOD2 can recognize both Gram types, since it is activated upon binding to muramyl dipeptide (MDP), a more common component of peptidoglycan [41, 42]. Interestingly, both NOD1 and NOD2 have recently been implicated in detection of parasites lacking peptidoglycan, indicating that these receptors can recognize a broader range of pathogens than was originally assumed $[43,44]$. Upon ligand-binding, NOD1 and NOD2 recruit the serine/threonine kinase RIPK2 (also known as RIP2) via CARDCARD interactions, eventually leading to the activation of $\mathrm{NF} \kappa \mathrm{B}[45,46]$. In addition, NOD1/2 stimulation also induces MAP kinase signaling [47]. Synergistically, with TLR activation, NOD1/2 signaling cascades induce the expression 


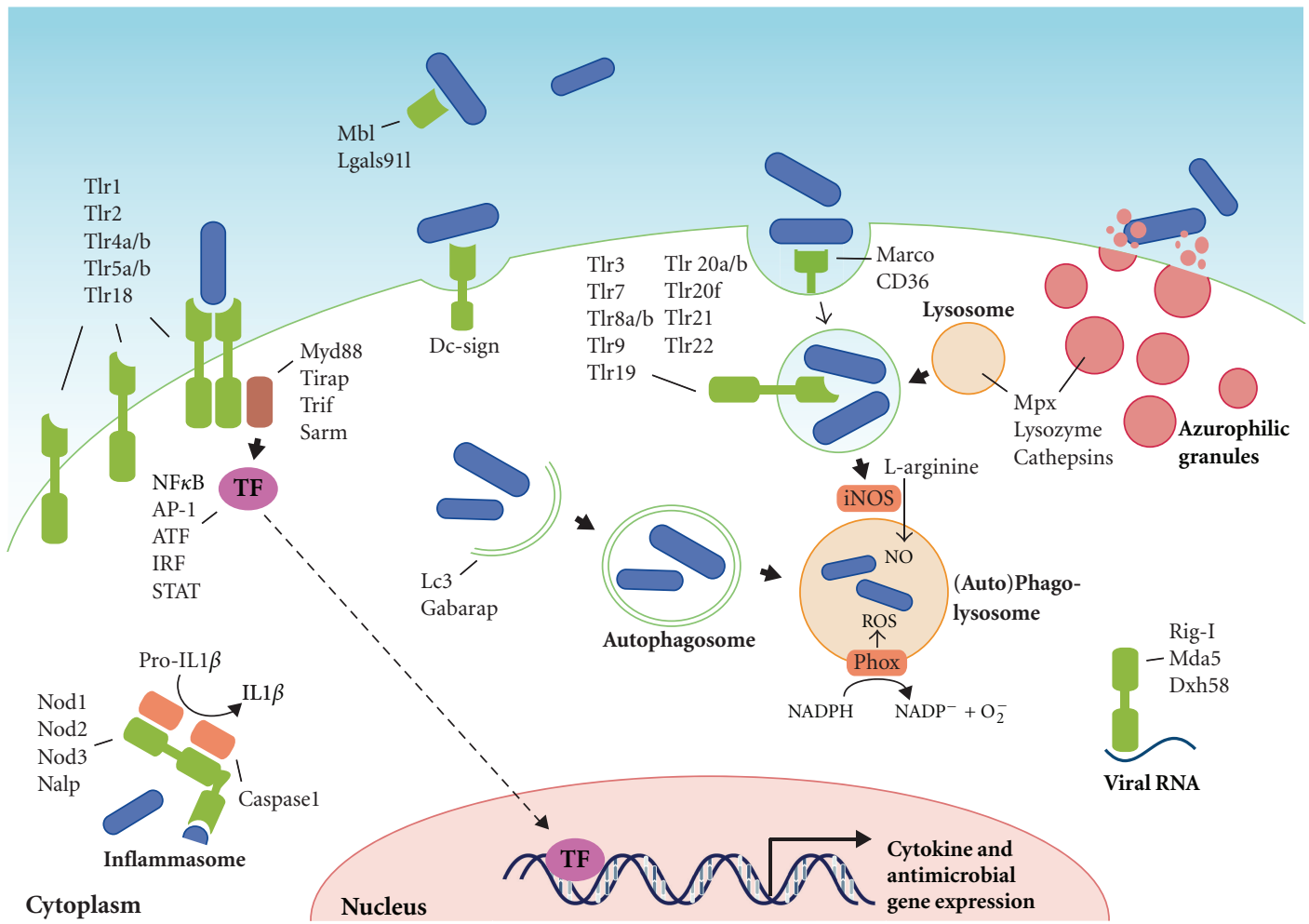

Figure 1: Pattern recognition receptors and effector mechanisms of the innate immune system. The localization of Tlrs on the cell surface or on endosomes is hypothetical and based on the known or proposed functions of their homologs in other fish or mammals. The ability of PRRs (depicted in green) to recognize PAMPs present on various types of microorganisms, like bacteria, viruses, and fungi, has been simplified here by depicting microorganisms as rod-like bacteria (in blue). PAMP recognition by PRRs leads to activation of transcription factors (TFs), which translocate to the nucleus and initiate transcription of cytokine genes, antimicrobial genes, and other immune-related genes. Defense mechanisms such as autophagy, ROS and NO production, and degranulation can be immediately activated upon microbial recognition, without de novo gene transcription.

of cytokines and chemokines, such as TNF, IL6, IL8, IL10, and IL12, as well as the production of antimicrobial peptides $[46,48,49]$.

Other NLRs, such as IPAF, NALP1, and NALP3, mainly function to create a multiprotein complex known as the inflammasome, in which they associate with an adaptor called ASC (apoptosis-associated speck-like protein containing a CARD) and with procaspase 1 [50]. Oligomerization of the proteins in an inflammasome via CARD-CARD interactions ultimately leads to the cleavage of procaspase 1 into its active form, caspase 1 , which is then available to catalyze the cleavage of accumulated pro-IL1 $\beta$ and pro-IL18 into their secreted forms, biological active IL1 $\beta$ and IL18 [40]. The NLR family member incorporated into these complexes determines which PAMPs and DAMPs are recognized by the inflammasome. A role for NALP3 has been established in the recognition of ATP [51], uric acid crystals [52], viral RNA [53], and bacterial DNA [54]. Both NALP1 and NALP3 share NOD2's ability to respond to MDP [55]. Furthermore, NALP1 can associate with NOD2 (Hsu 2008), showing a role for NOD2 in MDP-triggered IL1 $\beta$ activation, separate from its role as an inducer of proinflammatory gene expression.

Although the function of NLR family members in zebrafish is not widely studied, it is known that the canonical members of the mammalian NLR family, NOD1, NOD2, and
NOD3 (or Nlrc3) are conserved. Additionally, a subfamily of NLRs resembling the mammalian NALPs and a unique teleost NLR subfamily are present $[34,56]$. Confirmation of the antibacterial role of NOD1 and NOD2 in zebrafish was achieved by gene knockdown, resulting in higher bacterial burdens and decreased survival of embryos following Salmonella enterica infection [57]. Moreover, nod1/2 depletion significantly decreased expression of dual oxidase (DUOX), required for production of reactive oxygen species (ROS) [57]. These findings illustrate that the family of Nod-like receptors and their downstream signaling pathways are important for antibacterial innate immunity, both in mammals and in zebrafish.

2.3. RIG-I-Like Receptors. Another family of cytosolic PRRs, the RIG-I-like receptors (RLRs), consists of three members: RIG-I (retinoic acid-inducible gene I), MDA5 (melanoma differentiation-associated factor 5), and LGP2 (laboratory of genetics and physiology 2). All three members are $\mathrm{DExD} / \mathrm{H}$ box RNA helicases that can detect the presence of RNA from a broad range of viruses [58]. While expressed at low levels in most tissues, their expression is greatly increased upon viral infections or interferon (IFN) exposure [59, 60]. The RNA helicase domain of RLRs has the capacity to hydrolyze 
ATP and bind to RNA [61]. Furthermore, RIG-I and MDA5 contain a tandem of CARDs, which facilitate protein-protein interactions [60]. LGP2 lacks the two CARDs and is thought to function as a negative regulator of RIG-I and MDA5 signaling [62]. Following recognition of viral RNA, the CARDs of RIG-I and MDA5 become available for binding to a common mitochondrial signaling adaptor, IPS-1 or MAVS [63]. The subsequent signaling cascade culminates in the induction of transcription factors like interferon regulatory factor 3 (IRF3), IRF7, and NFאB [64]. Activation of these transcription factors leads to the production of type I IFN, which binds to the IFN receptor to initiate expression of interferon-stimulated genes (ISGs) [65]. Amongst these ISGs are antiviral proteins, immune-proteasome components, all three RLRs, members of the TLR family, transcription factors like IRF7, and various proinflammatory cytokines and chemokines [65]. As such, the RLR-induced pathway works cooperatively with TLR signaling to prepare the cell for elimination of viral infections [58].

Zebrafish homologs of RIG-I, MDA5, and DXH58 were identified in a genome search [66]. However, in silico analysis of the predicted proteins revealed that the domain distribution differs between humans and zebrafish [66]. For instance, whilst human RIG-I contains two CARDs, one $\mathrm{DExD} / \mathrm{H}$ domain and a Helicase $\mathrm{C}$ domain, zebrafish RIGI consists of a single CARD and a $\mathrm{DExD} / \mathrm{H}$ domain [66]. Whilst functional studies of the RLR pathway are scarce, it is clear that zebrafish and other teleosts possess a strong antiviral IFN system, which shares a common evolutionary origin with mammals $[67,68]$. The mitochondrial RLR adaptor, IPS-1/MAVS, was recently cloned from salmon and zebrafish, and overexpression in fish cells led to a constitutive induction of ISGs [68]. Furthermore, MITA, another adaptor functioning downstream of IPS-1/MAVS and upstream of Tank-binding kinase 1 (TBK1), was cloned from crucian carp (Carassius auratus) and shown to activate zebrafish IFN promoter gene constructs, dependent on IRF3 or IRF7 [69].

2.4. Scavenger Receptors. Scavenger receptors are a large family of transmembrane cell surface receptors, present on macrophages, dendritic cells, mast cells [70], and some endothelial and epithelial cell types [71]. Although originally defined for their role in uptake of low-density lipoproteins (LDL), they are now known to act as PRRs for a wide variety of PAMPs, like LPS, LTA, CpG DNA, yeast zymosan, and microbial surface proteins [72]. Commonly, PAMP binding to a scavenger receptor will induce the cell to directly phagocytose the pathogen [73]. Upregulation of scavenger receptor expression via TLR signaling can be a mechanism to increase phagocytic activity [74]. Moreover, scavenger receptors can also contribute to cytokine production as coreceptors for TLRs [75, 76]. Some of the C-type lectins, discussed below, also display scavenger receptor activity.

Based upon their multidomain structure, scavenger receptors are divided into eight subclasses (A-H) (Murphy 2005). Subclasses A and B are the most extensively studied, but members from other subclasses have also been shown to recognize bacterial PAMPs [72]. SR-A, the founding member of subclass A, functions as a phagocytic receptor for bacterial pathogens like Staphylococcus aureus, Neisseria meningitides, Streptococcus pneumonia, and Escherichia coli [77-79]. Macrophage receptor with collagenous structure (MARCO), another subclass A member with established PRR activity [80], functions as a phagocytic receptor for S. pneumonia [81] and N. meningitidis [82]. MARCO was shown to cooperate with TLR2 to trigger macrophage cytokine responses to the mycobacterial cell wall glycolipid trehalose dimycolate (TDM) and Mycobacterium tuberculosis [83]. CD36, the most prominent member of subclass $\mathrm{B}$, is a sensor for LTA and diacylated lipopeptide (MALP-2) and also acts as a coreceptor for TLR2 [75]. CD36-mediated phagocytosis of $S$. aureus was shown to be required for initiation of TLR2/6 signaling [84]. SR-BI (or CLA-1), also in subclass $\mathrm{B}$, can bind to LPS and was implicated in phagocytosis of both Gram-negative and Gram-positive bacteria [85]. As well as their antibacterial roles, CD36 and SR-BI are also known for increasing the pathogenesis of malaria and hepatitis $\mathrm{C}$ virus (HCV). CD36 can function as a receptor for erythrocytes that have been parasitized by Plasmodium falciparum, adhering these cells to the venular endothelium of various organs (Pluddemann 2007). Furthermore, SR-BI is used by Plasmodium sporozoites and HCV as an entry site into hepatocytes [72].

Many homologs of the mammalian scavenger receptor family can be identified in the zebrafish genome, but a systematic analysis is still awaited. A zebrafish homolog of human MARCO was identified as a specific marker for macrophages and dendritic cells from adult zebrafish [86], and this gene is also myeloid specific in zebrafish embryos [87]. Expression of the $c d 36$ gene was upregulated after exposing zebrafish to haemorrhagic septicemia rhabdovirus [88]. In contrast, $c d 36$ expression was downregulated by Mycobacterium marinum infection in adult zebrafish and larvae [22].

2.5. C-Type Lectins. The C-type lectin receptors (CLRs) are a large family of carbohydrate-binding proteins that are highly conserved amongst mammals [89]. The diversity of the CLR family is illustrated by the fact that up to 17 groups are present in vertebrates, with some consisting of soluble serum proteins, whilst others consist of transmembrane proteins. These are mainly expressed in myeloid cells (macrophages and dendritic cells) but also in natural killer cells [90, 91]. The best known CLR in serum is mannose-binding lectin (MBL), a member of the collectin class, which binds to a variety of sugar moieties present on viruses, bacteria, fungi, and protozoa and activates the complement system [92]. In terms of their function as PRRs, the transmembrane CLRs that are expressed on myeloid cells are the most interesting. Transmembrane CLRs can be divided into two groups: the mannose receptor family and the asialoglycoprotein receptor family [93]. CLRs recognize pathogens mainly via ligand binding to mannose, fucose, and glucan carbohydrate structures, which means that together they are capable of recognizing most classes of human pathogens [93]. Like scavenger receptors, CLRs can act as phagocytic receptors 
for nonopsonized bacteria, leading to their destruction in acidified phagolysosomes [73]. The best-studied member of the asialoglycoprotein receptor family is Dectin-1, which mediates phagocytosis of yeast and the yeast-derived protein zymosan [94]. Phagocytosis induced by CLRs like Dectin1 is not only important for the lysosomal breakdown of pathogens, but also for antigen presentation $[95,96]$. Besides their role in phagocytosis, CLRs can directly induce gene expression upon carbohydrate recognition. PAMP recognition by Dectin-1, Dectin-2, and macrophage-inducible Ctype lectin (Mincle) ultimately leads to activation of $\mathrm{NF} \kappa \mathrm{B}$ [97-99]. Where Dectin-1 associates with the kinase Syk to activate NF $\kappa B$ [100], Dectin-2 and Mincle are dependent on Fc receptor $\Upsilon$-chain as an adaptor molecule $[98,99]$. Other CLRs, for example, DC-specific ICAM3-grabbing nonintegrin (DC-SIGN), induce specific gene expression profiles upon pathogen recognition by modulating TLR signalling [93]. When DC-SIGN recognizes mannose or fucose moieties on pathogens such as Mycobacteria, HIV1, measles virus, and Candida albicans, it activates a Raf-1dependent signaling pathway that modulates TLR-induced $\mathrm{NF} \kappa \mathrm{B}$ activation, increasing the production of IL8 and antiinflammatory IL10 production [101].

Only a few homologs of CLRs have been described in zebrafish. A homolog of the complement activating mannose-binding lectin (MBL) was associated with resistance against Listonella anguillarum [102]. Expression of another soluble lectin, lgals91l, is enriched in zebrafish embryonic myeloid cells and is dependent on the Spi1/Pu.1 transcription factor that plays a crucial role in myeloid cell development in vertebrates [87]. A membrane type collectin, CL-P1 (collectin placenta 1), was shown to be involved in vasculogenesis during zebrafish embryogenesis [103]. In humans, CL-P1 is mainly expressed on vascular endothelial cells and has been shown to act as a scavenger receptor mediating the phagocytosis of bacteria and yeast [104]. A putative homolog for DC-SIGN has recently been proposed and is upregulated in immune-related tissues following infection by Aeromonas anguillarum [105]. Finally, putative homologs for the mammalian C-type lectin NK cell receptors have been identified in zebrafish and are differentially expressed on cells from the myeloid and lymphoid lineages [106].

\section{Effector Mechanisms of the Innate Immune Response in Zebrafish}

While the adaptive immune system requires several days before reacting to invading microbes, the innate immune system consists mostly of defenses that are constitutively present and activated immediately upon infection (Figure 1). The general inflammatory response is a crucial innate defense mechanism. A state of inflammation is necessary for proper function of host defenses, since it focuses on circulating immune cells and antimicrobial components of the plasma at the site of infection. Below, we focus on the effector mechanisms involved in the cell-mediated part of the innate immune response. In addition, soluble serum proteins, including complement factors and other acutephase proteins, make an important contribution to the innate defenses, and strong induction of their encoding genes has been observed in adult and embryonic zebrafish infection models [13, 36, 38, 107-109].

3.1. Secreted Peptides and Lipid Mediators of the Innate Immune Response. Cytokines, including interleukins, chemokines, and interferons, are small secreted proteins that steer the host's immune system into a cytotoxic, humoral, cell-mediated, or allergic response [110]. Since this paper focuses on innate immunity, we will mainly discuss the cytokines produced by or acting on phagocytic cells. A distinction can be made between cytokines that promote a state of inflammation and cytokines that are anti-inflammatory. The main proinflammatory cytokines produced by phagocytes are TNF $\alpha$, IL1 $\alpha$, IL1 $\beta$, IL6, and IL 8 [110]. TNF- $\alpha$ is processed as a membrane-bound protein and, when required, the active soluble factor is cleaved off by the TNF- $\alpha$ converting enzyme (TACE) [111]. Similarly, IL1 $\alpha$ and IL1 $\beta$ are synthesized as inactive precursors that are only secreted as active cytokines after inflammasomemediated cleavage by caspase 1 [112]. The most potent antiinflammatory cytokine in humans is IL10, which deactivates the proinflammatory cytokine production by macrophages and $\mathrm{T}$ cells [113]. The IL10/IL12 balance, maintained by cells of the innate immune system, determines whether adaptive immunity polarizes towards a Th1 (promoted by IL12) or Th2 response. A Th1 response, which activates the bactericidal activities of macrophages, is the most important for controlling intracellular pathogens. The single type II IFN, IFN $\gamma$, is also required for activating macrophage bactericidal functions, while type I IFNs (IFN $\alpha$ and IFN $\beta$ ) and type III IFN (IFN $\lambda$ ) function in mounting antiviral responses. Finally, eicosanoid lipid mediators also promote (e.g., prostaglandins and leukotrienes) or inhibit (e.g., lipoxins) inflammation, thus synergizing with or antagonizing cytokine functions.

Many of the cytokine subfamilies are conserved between zebrafish and mammals [34]. However, there has been extensive expansion and diversification of members of the chemokine gene family in zebrafish, and their specific functions are yet to be determined [114]. Several of the main cytokines, like IL1 $\beta$, IL6, and IL10, have been cloned and characterized [115-117]. Furthermore, the zebrafish homolog of interleukin 10 receptor 1 (IL10R1) has recently been identified and seems to contain all the protein domains that are required for its function in anti-inflammatory signaling [118]. The proinflammatory chemokine IL8 (CXCL8) and it receptors, CXCR1 and CXCR2, are also conserved between mammals and zebrafish [119]. In addition, a second IL8/CXCL8 lineage has been identified in both zebrafish and common carp (Cyprinus carpio), and the chemotactic properties of carp IL8/CXCL8 molecules of both lineages were demonstrated by in vitro chemotaxis assays using carp leukocytes [120]. Both pro- and anti-inflammatory cytokines are upregulated upon infection of zebrafish embryos with pathogens such as S. typhimurium [13], P. aeruginosa [121], and E. tarda $[122,123]$. 
The role of TNF during Mycobacterium marinum infection of zebrafish embryos was studied by knockdown analysis

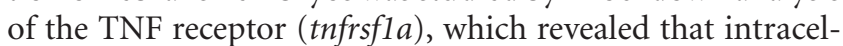
lular bacterial burdens, granuloma formation, and necrotic death of macrophages are increased in the absence of TNF signaling [124]. The importance of TNF signaling during M. marinum infection was further illustrated when the same model was used to show that a strict balance between proinflammatory TNF and anti-inflammatory lipoxins is vital for control of mycobacterial infections, with either too much or too little TNF expression leading to a more severe outcome of the disease [1]. Another study using the zebrafish model indicates that TNF- $\alpha$ is a potent activator of endothelial cells, leading to the production of chemokines, whilst it has little effect on the activation status of phagocytes [125]. This suggests that fish TNF- $\alpha$ mainly functions in the recruitment of leukocytes to the site of infection, rather than activating them.

The three IFN groups present in humans are not conserved unambiguously in zebrafish and other fish species. The type II group of IFNs in zebrafish consists of IFN $\gamma 1$ and IFN $\gamma 2$ [126]. Expression levels of the corresponding genes did not change upon infection of zebrafish embryos with $E$. coli or $Y$. ruckeri, but was increased by M. marinum infection $[126,127]$. Viral infection induced their expression in adult zebrafish but not in embryos [126]. IFN $\gamma 1$ and IFN $\gamma 2$ were shown to bind to different receptor complexes, and Janus kinase 2a (Jak2a), but not Jak2b, was shown to be required for intracellular transmission of the IFN $\gamma$ signal. Two groups of antiviral IFNs, named IFN $\phi 1$ and IFN $\phi 2$, exist in zebrafish, and structural analysis showed that these are evolutionarily closer to type I than to type III human IFNs $[34,67,128]$. IFN $\phi 1$ and IFN $\phi 2$ signal via distinct receptor complexes [67, 129]. All zebrafish IFN $\phi$ genes induce the expression of genes that are predicted to be involved in antiviral activities [67].

3.2. Phagocytosis, Autophagy, and Lysosomal Destruction. Internalization of microorganisms is triggered when they are recognized by phagocytic receptors, mainly by scavenger receptors discussed above. This type of direct phagocytosis is termed nonopsonic phagocytosis, while opsonic phagocytosis relies on host-derived proteins that coat the surface of the microbe thereby enhancing phagocytosis efficiency. Opsonins include complement fragments, most notably C3b, which are recognized by complement receptors [130]. Mannose binding lectin, which can initiate C3b formation, and antibodies that bind to Fc receptors ( $\mathrm{IgG}$ ) or that activate complement (IgM) are also considered opsonins. Regardless of which receptor initiates the process, phagocytosis requires the activation of kinases and Rab GTPases that control alterations in the phospholipid membrane and remodeling of the actin cytoskeleton [131]. In macrophages, fusion of the resulting vesicle with early and late endosomes will decrease the $\mathrm{pH}$ of the immature phagosome and alter the proteins present on its membrane. Ultimately, maturing phagosomes turn into phagolysosomes when lysosomes fuse with them, mixing their contents [132]. Lysosomes are highly acidic endocytic vesicles ( $\mathrm{pH}<5.5)$, containing active proteases and lipases, and hydrolytic enzymes such as cathepsin D [133]. In addition, phagolysosomes also contain bactericidal peptides (defensins) and have the ability to generate toxic oxidative compounds that help microbial degradation [134]. Most of our knowledge about phagosome maturation comes from studies of phagocytosis in macrophages, and much less is known about phagosome maturation in neutrophils. While macrophage phagosomes fuse with endosomes and lysosomes, neutrophil phagosomes obtain their bactericidal properties by fusing with secretory vesicles and granules $[135,136]$. In contrast to phagosome maturation in macrophages, neutrophil phagosomes do not acidify in order to become microbicidal $[135,136]$.

Many intracellular pathogens, like M. tuberculosis, S. typhimurium, and Legionella pneumophila, have evolved the ability to prevent phagosome maturation in macrophages and survive inside these vesicles [137]. To a certain extent, such pathogens can also withstand the hostile environment of the (phago)lysosome. Other pathogens like Listeria monocytogenes, Francisella tularensis, and many viruses can escape the phagosome and enter the cytosol [138]. Mycobacterium marinum, a pathogen studied extensively in zebrafish to model human tuberculosis, can survive inside phagosomes but also escape into the cytosol and spread to neighbouring cells by actin-based motility [139, 140]. Phagosomal escape has also been observed for the human pathogen $M$. tuberculosis and is dependent on a virulence factor, the ESX-/RD1 secretion system, shared by all pathogenic mycobacteria [141]. Together, these data indicate that host cells face numerous pathogens that have developed multiple strategies to avoid the pathway of phagolysosomal degradation. To counter such threats, cells may use autophagy to clear microbes and microbe-containing vesicles from the cytosol. Autophagy is well known as a metabolic process that recycles nutrients by degrading intracellular organelles and proteins. Only recently, it has been recognized that autophagy also plays an important role in the innate immune response against intracellular pathogens [142]. Autophagy is initiated when an autophagosomal isolation membrane is formed around its target, enclosing it entirely in a double-membrane vesicle. This process relies on class III phosphatidylinositol 3-kinase (PI3-kinase) and autophagyrelated genes (Atgs), such as Atg6 (or Beclin-1) [143]. The hallmark of autophagosomes is the presence of Atg8 (or LC3) in their membranes, which is essential for membrane elongation [144]. Similar to a maturing phagosome, the autophagosome also fuses with lysosomes to achieve its degradative properties [145]. In addition, autolysosomes acquire unique antimicrobial properties due to the function of autophagic adaptor protein p62, which delivers cytosolic components to autolysosomes where they are processed into potent antimicrobial peptides [146]. As reviewed elsewhere [147], pathogen-targeted autophagy can be induced by several TLRs and NLRs, TNF- $\alpha, \mathrm{NF} \kappa \mathrm{B}$, and many other immune-related signalling molecules.

The transparency of zebrafish embryos and availability of fluorescent macrophage and neutrophil reporter lines allow for study of the process of phagocytosis in great detail 
[7, 148-150]. It was recently shown that zebrafish embryonic macrophages efficiently engulf $E$. coli bacteria from bloodand fluid-filled cavities, while neutrophils are hardly capable of phagocytosing bacteria present in fluids [150]. However, neutrophils did prove to be highly phagocytic when moving over bacteria present on tissue surfaces. This shows that the type of immune cell that clears an infection not only depends on the PAMPs present on the invading microbe, but also on the characteristics of the infection site. An in vivo phagocytosis assay was used to show that functions of Wasp1, Wasp2, Abi2, and cofilin regulator $14-3-3 \zeta$ (Ywab) in bacterial phagocytosis are conserved in zebrafish [151]. The recent generation of a transgenic zebrafish line with GFP-tagged LC3 has enabled in vivo visualization of the interactions between microbes and this core component of the autophagy machinery [152]. The importance of autophagy in the innate immune response of zebrafish remains to be studied, but we have shown that LC3-labeled structures accumulate around M. marinum infection sites in zebrafish embryos (Figure 2). Furthermore, autophagy-related genes were induced in adult zebrafish infected with Citrobacter freundii and zebrafish embryos infected with S. typhimurium $[37,153]$.

3.3. Oxidative Defenses in Leukocytes. In several systems, it has been shown that neutrophils are the first immune cells to arrive at the site of infection or wounding. They facilitate their migration by exocytosing granules that contain metalloproteinases and other enzymes that degrade the extracellular matrix [154]. Upon recognition of pathogens, neutrophils release their antimicrobial granules, called azurophils, into phagosomes or the extracellular environment $[155,156]$. Azurophils are packed with acidic hydrolases and antimicrobial proteins, such as lysozyme, cathepsins, and myeloperoxidase (MPO) [157]. The primary function of MPO is to react with hydrogen peroxide $\left(\mathrm{H}_{2} \mathrm{O}_{2}\right)$, which subsequently oxidates chloride, tyrosine, and nitrite to form hypochloric acid $(\mathrm{HOCl})$, tyrosine radicals, and reactive nitrogen intermediates [158]. These highly reactive chemicals attack the surface membranes of microbes. Additionally, microbes can be bound by neutrophil extracellular traps (NETs), which are fibrous networks of granule proteins and chromatin released by neutrophils [159].

While MPO is mostly produced in neutrophils, all professional phagocytes produce high levels of reactive oxygen species (ROS), including superoxide, $\mathrm{H}_{2} \mathrm{O}_{2}$, and hydroxyl radicals, produced by the enzymes NADPH oxidase (NOX) and dual oxidase (DUOX) [160]. The NOX of phagocytes (Phox) is only activated upon exposure to microorganisms or other pro-inflammatory stimuli [161]. When active, Phox is located in the phagosomal membrane and catalyzes the respiratory burst, which consists of the large-scale production of ROS that helps degrade phagocytosed microbes by nonspecifically oxidizing protein, DNA, lipid, and carbohydrate [162]. $\mathrm{H}_{2} \mathrm{O}_{2}$ produced during the respiratory burst can also function as a substrate for MPO activity. The oxidative enzyme DUOX may even combine the two functions, by generating $\mathrm{H}_{2} \mathrm{O}_{2}$ as a substrate for its own peroxidase domain [160].
Nitric oxide (NO) is produced from the amino acid L-arginine by nitric oxide synthase (NOS) enzymes and functions as a signaling molecule in numerous biological processes as well as having antimicrobial activity [163]. There are two constitutively expressed NOS enzymes, neuronal NOS (nNOS or NOS1) and endothelial NOS (eNOS or NOS3), and one inducible NOS (iNOS or NOS2) that is important in innate immunity. Regulation of NOS2 plays an important role in the inflammatory response, and many cells of the immune system are capable of producing NO $[164,165]$. NO has cytostatic and cytotoxic antimicrobial effects when high amounts are excreted by immune cells into mammalian tissues, most likely via reactive nitrogen species (RNS) which are generated when NO interacts with $\mathrm{O}_{2}$ [166]. These RNS subsequently lead to lipid peroxidation, DNA damage, oxidation of thiols, and nitration of tyrosine residues [167]. It has recently been shown that Nos2a, the zebrafish homolog of NOS2, is also required for the expansion of hematopoietic stem cells and progenitor cells during infection, leading to increased numbers of the required immune cells [168]. This discovery further adds to the importance of NOS2 in the inflammatory response.

The oxidative defense mechanisms need to be tightly controlled, since high levels of reactive chemicals like ROS and RNS cause tissue damage at sites of infection. Therefore, the resolution phase of inflammation is critical in order to restore the tissue to its normal state and prevent chronic inflammation. The molecules produced during oxidative defenses are often self-limiting and help initiate resolution of inflammation by inducing neutrophil apoptosis [160, 169]. Furthermore, iNOS-induced NO production can be countered by activation of arginase (ARG), which depletes the substrate for iNOS by converting L-arginine to the harmless compounds urea and L-ornithine, thus creating conditions more favorable for wound healing $[163,170]$.

The zebrafish homolog of MPO, officially named MPX, is specifically expressed in neutrophils during embryonic development. Transgenic reporter lines driven by the $m p x$ promoter have made the zebrafish a highly suitable model organism to study neutrophilic inflammation $[8,171]$. In fact, using one of these lines, it was demonstrated for the first time that $\mathrm{H}_{2} \mathrm{O}_{2}$ produced in the context of wounding not only functions as an antiseptic compound, but also forms a gradient that is required for rapid attraction of leukocytes [172]. However, this $\mathrm{H}_{2} \mathrm{O}_{2}$ gradient is only generated at wounds and does not occur at infected tissues [173]. The formation of this $\mathrm{H}_{2} \mathrm{O}_{2}$ gradient was shown to be dependent on the oxidase activity of Duox. The Src family kinase Lyn has been identified as the redox sensor that mediates neutrophil migration towards the wound [174]. The innate immune function of Duox and the importance of ROS in zebrafish were further established by studies showing that knockdown of Duox impaired the ability of zebrafish larvae to control enteric Salmonella infections [175]. It has also been shown that zebrafish Phox is important in controlling the in vivo growth of the pathogenic fungus Candida albicans [176]. A 5,5-dimethyl-l-pyrroline N-oxide- (DMPO-) based immunospin trap technique has been adopted for in situ detection of ROS production in zebrafish embryos [177]. 


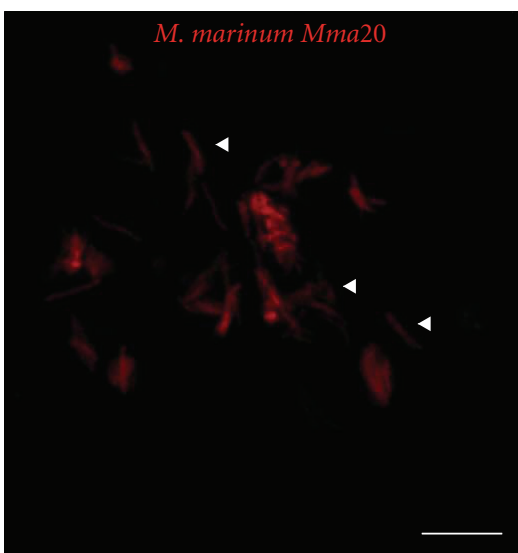

(a)

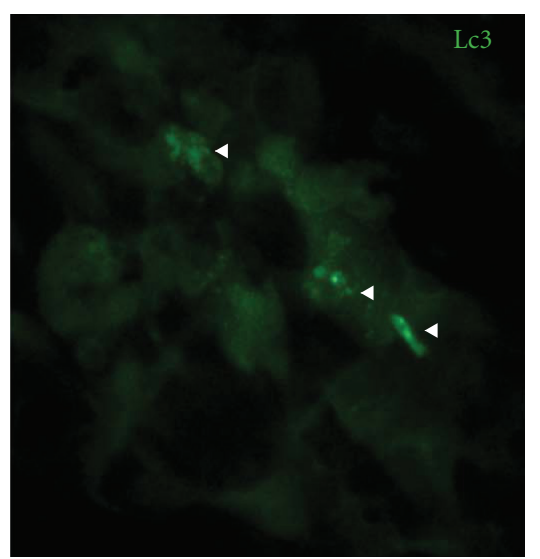

(b)

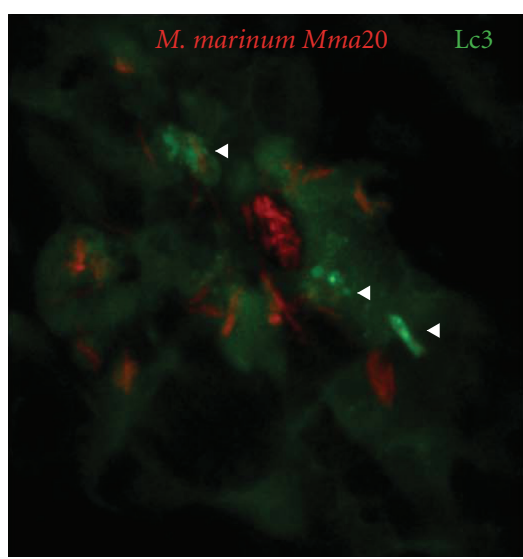

(c)

FIgURE 2: In situ detection of autophagy by Lc3 accumulation. CMV::LC3-GFP transgenic [15] zebrafish embryos (28 hpf) were injected into the caudal vein with 200 colony-forming units (CFU) of M. marinum Mma20 expressing a pMST3::mCherry vector. Confocal images were taken of a tail region of the developing larva at 3 days after infection ( $3 \mathrm{dpi}$ ), a point at which the M. marinum infection (a) has been established. Low levels of Lc3-GFP signal (b) can be observed throughout the cells, whilst brighter regions (indicated by arrowheads) are only observed upon Lc3 accumulation and formation of autophagic membranes associated with bacteria (c). Scale bar: $10 \mu \mathrm{m}$.

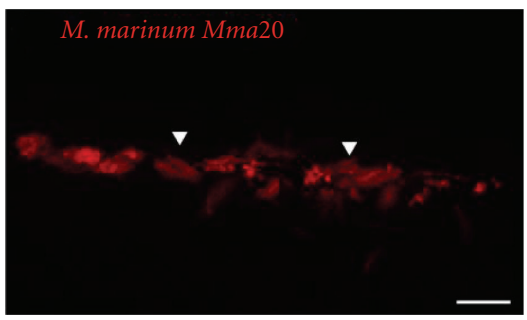

(a)

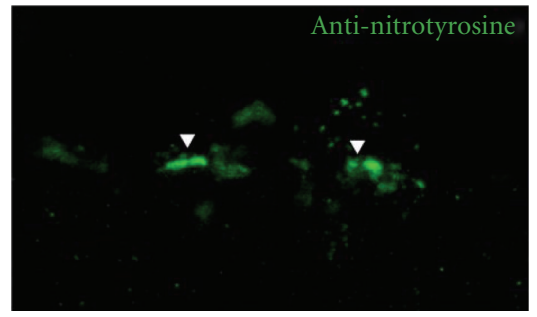

(b)

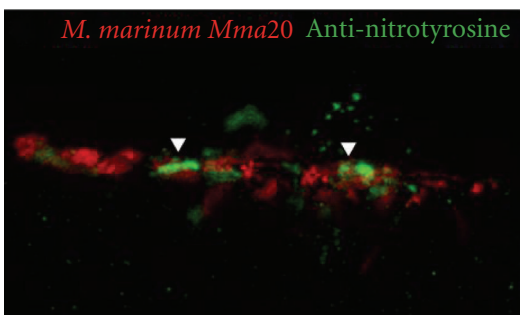

(c)

FIGURE 3: In situ detection of reactive nitrogen species. Wild-type zebrafish embryos (Albino; 28 hpf) were injected into the caudal vein with 200 colony-forming units (CFU) of M. marinum Mma20 expressing a pMST3::mCherry vector. Confocal images were taken of a tail region of the developing larva at 3 days after infection ( $3 \mathrm{dpi}$ ), a point at which the M. marinum infection (a) has been established. Embryos were fixed in $4 \%$ paraformaldehyde at $3 \mathrm{dpi}$, and immunohistochemistry was performed, using an antinitrotyrosine antibody that detects tissue nitration (b) [21]. Colocalization (c) between bacteria and extensive tissue nitration can be observed at this time point. Scale bar: $10 \mu \mathrm{m}$.

DMPO is a chemical substrate that binds to reactive oxygen, which can later be detected with an anti-DMPO antibody. This protocol detects the build-up of the conjugated product, thereby showing a cumulative ROS production. Furthermore, a respiratory burst assay has been developed for zebrafish embryos, which was used to demonstrate that macrophages and neutrophils are the ROS-producing cells in zebrafish [178]. A similar method is available to image the production of NO in zebrafish embryos, using a diaminofluorescein probe that only becomes fluorescent in the presence of NO [179]. As mentioned before, nitration of tyrosine residues is a hallmark of $\mathrm{NO}$ production. Forlenza et al. (2008) used an antinitrotyrosine antibody on common carp tissue to visualize the tissue nitration that occurs at sites of Trypanoplasma borreli infection [21]. We used the same antibody for immunohistochemistry on zebrafish embryos to visualize the production of RNS in response to $M$. marinum infection (Figure 3 ). This technique also visualizes the nitrosative stress that the host tissue suffers upon release of RNS. The resolution of inflammation that should prevent tissue damage following such stresses has also been studied in zebrafish. This has led to new insights on the mechanisms underlying resolution, including apoptosis and retrograde chemotaxis of neutrophils, with the oxygen-sensing transcription factor hypoxia-inducible factor- $1 \alpha($ Hif- $1 \alpha)$ playing a role in the control of these mechanisms $[171,180]$.

\section{Gene Expression Programs Reflecting Innate Immune Responses}

4.1. Genome-Wide Expression Profiling. The availability of the zebrafish genome sequence facilitates the use of microarray and deep sequencing techniques for genome-wide expression profiling. Zebrafish embryos and larvae are useful for in vivo analysis of gene expression profiles upon infection, since large numbers can be pooled to level out individual variation. However, pooling should be done with caution, and it is advisable to verify conclusions by analysis at the single-embryo level [123]. A protocol has been developed for single embryo RNA isolation that gives sufficient RNA 
TABLE 1: Transcriptome profiling studies on infection models in adult and embryonic zebrafish.

\begin{tabular}{lccc}
\hline Bacterial species & Strain & Infection model & Reference \\
\hline Mycobacterium marinum & M; E11 & Adult (IP) & Meijer et al.*[182] \\
Mycobacterium marinum & Mma20; E11 & 28hpf (CV); Adult (IP) & Van der Sar et al. [22] \\
Mycobacterium marinum & M; E11 & Adult (IP) & Hegedus et al.* [107] \\
Salmonella enterica serovar & SL1027; & & \\
Typhimurium & LPS derivative & 28hpf (CV) & Stockhammer et al.** [13] \\
(Salmonella typhimurium) & SF1592 $(R a)$, & Adult (IP) & Wu et al. [183] \\
Streptococcus suis & HA9801 & & \\
Salmonella enterica serovar & SL1027; & 28hpf (CV) & Ordas et al.** [38] \\
Typhimurium & LPS derivative & & \\
Salmonella typhimurium) & SF1592 $(R a)$, & 28hpf $(\mathrm{CV})$ & Van Soest et al. [123] \\
Edwardsiella tarda & FL6-60 & Adult (IM) & Lu et al. [153] \\
Citrobacter freundii & Not specified & & \\
\hline
\end{tabular}

* and ${ }^{* *}$ : these studies used the same samples but applied microarray analysis and deep sequencing, respectively. (IP): intraperitoneal; (CV): caudal vein; (IM): immersion.

for microarray or RNA sequencing [181]. Expression profiling can be done either at whole organism level or on FACS-sorted immune cells from transgenic lines. The latter approach was used to determine the transcriptional signature of early myeloid cells [87]. Microarray analysis of zebrafish adults and embryos infected with various pathogens has provided insights into the transcriptome during infection and has provided leads for further functional studies (Table 1). The transcriptional response of both zebrafish embryos and adults showed clear conservation with host responses detected in other vertebrate models and human cells. Genes that were induced upon infection included receptors involved in pathogen recognition, signaling intermediates, their downstream transcription factors (like $\mathrm{NF} \kappa \mathrm{B}$ and AP-1), and inflammatory mediators. Furthermore, these studies led to the identification of novel immune responsive genes and infection markers, for example, the DNA-damageregulated autophagy modulator 1 gene ( $\operatorname{ram} 1)$, which was identified in a knockdown study of Traf6, a central intermediate in TLR and TNF receptor signaling [37].

4.2. Comparison of Gene Expression Profiles Induced by Different Bacterial Pathogens. To illustrate the similarities and differences in the innate immune response against different bacterial pathogens, Figure 4 shows a comparison of the gene expression profiles of zebrafish infected with Edwardsiella tarda, S. typhimurium, and M. marinum. E. tarda is a Gram-negative, naturally occurring fish pathogen that belongs to the Enterobacteriaceae family. Inside its host, $E$. tarda is able to resist complement activity and can survive inside macrophages [184]. It causes a progressive disease when injected into the caudal vein of 28 hours after fertilization (hpf) embryos, leading to mortality within 2 days after infection (dpi) [123]. S. typhimurium (short for S. enterica serovar Typhimurium), also belonging to the Gramnegative Enterobacteriaceae family, causes salmonellosis in a broad range of hosts. S. typhimurium is a facultative intracellular species that can survive within phagocytic and nonphagocytic cells. Following internalization, it survives and replicates in a modified phagosome, known as the Salmonella-containing vacuole. Like E. tarda, injection of S. typhimurium into the caudal vein at $28 \mathrm{hpf}$ leads to a progressive disease which leads to mortality of the embryo during the first 30 hours after infection (hpi) $[13,185]$. In contrast, M. marinum injection at the same stage leads to a chronic infection that persists during larval development. $M$. marinum is a natural pathogen of teleost fish and a close relative of $M$. tuberculosis, the causative agent of tuberculosis in humans. Mycobacteria have a thick, waxy, acid-fast staining cell wall containing characteristic lipids that are important for virulence. Both $M$. marinum and $M$. tuberculosis have the ability to replicate inside macrophages, eventually causing them to undergo apoptosis. Dependent on secreted virulence factors that are conserved between M. marinum and M. tuberculosis, other macrophages are attracted to the initial infection site. These become infected by phagocytosing the apoptotic remains, which ultimately leads to the formation of a granuloma [186]. Using the zebrafish embryo model, Ramakrishan et al. have provided new insights demonstrating the importance of the innate immune system to control $M$. marinum infection during early stages of pathogenesis $[1,2,124,187,188]$.

Complementary to previously reported transcriptome data (Table 1), here we present new data comparing the gene expression profiles induced by E. tarda, S. typhimurium, and M. marinum under similar conditions (Figure 4). We injected 200 colony-forming units (CFUs) of each pathogen into the caudal vein of $28 \mathrm{hpf}$ zebrafish embryos and analyzed the response at $8 \mathrm{hpi}$. Since M. marinum develops a chronic infection, we also sampled at $4 \mathrm{dpi}$, a time point at which granulomas are present. Finally, we compared the transcriptome profile of the embryonic samples with data from a previous study, in which adult zebrafish were infected with the same strain of M. marinum [22].

The two progressive Gram-negative pathogens, E. tarda and S. typhimurium, induced a strong early immune response at $8 \mathrm{hpi}$, while the chronic M. marinum infection hardly induced any response at this time point. At $4 \mathrm{dpi}$, the transcriptome profile of $M$. marinum-infected embryos did 


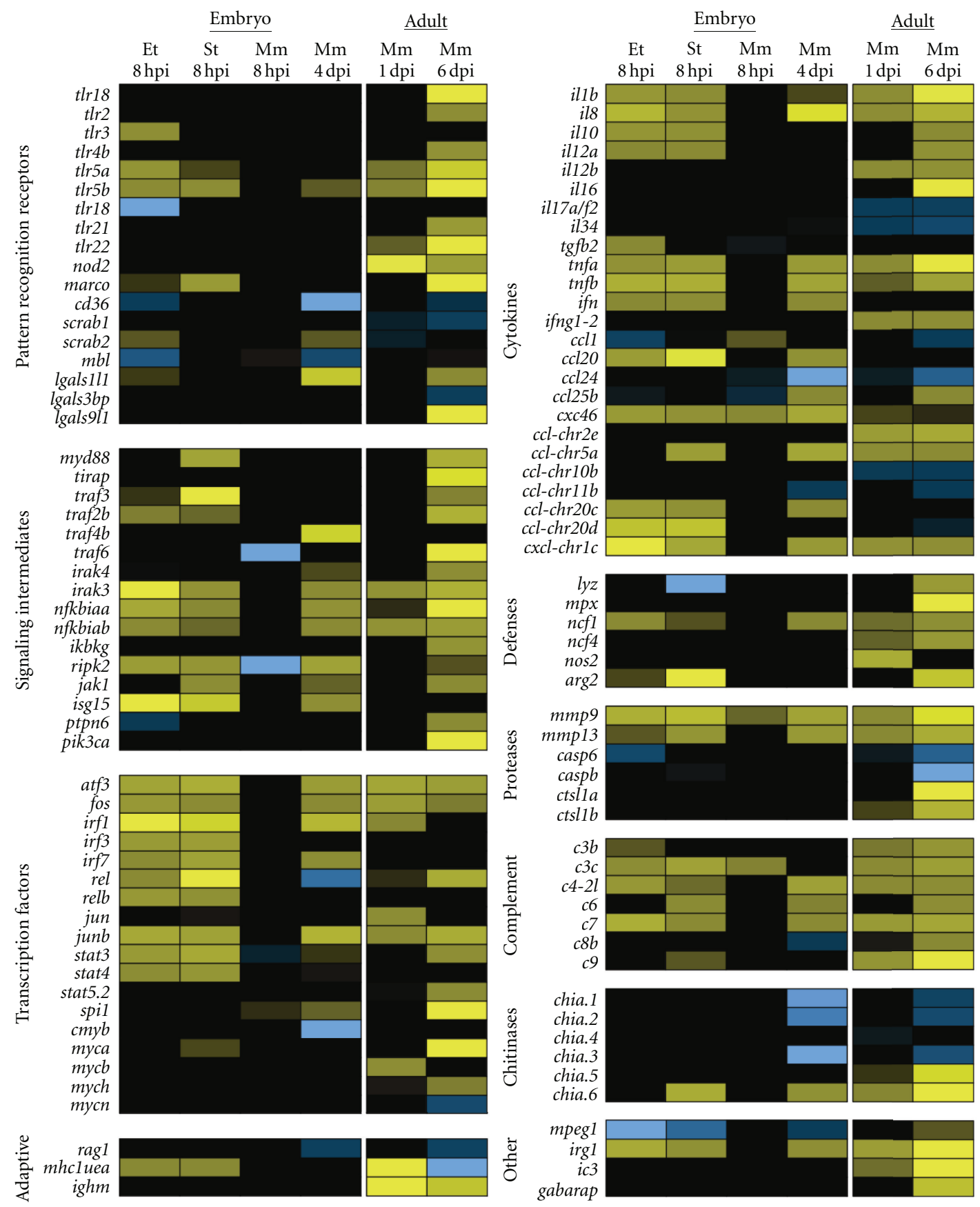

FIgURE 4: Comparison of the zebrafish innate immune response to different bacterial pathogens. Gene expression profiles of zebrafish embryos and adults infected with E. tarda FL6-60 (Et), S. typhimuriumSL1027(St), and M. marinum Mma20 (Mm) are depicted in a heat map. Embryos were infected with $200 \mathrm{CFU}$ of each pathogen into the caudal vein at $28 \mathrm{hpf}$ and snap frozen individually at $8 \mathrm{hpi}$ for $E$. tarda and S. typhimurium, and at 8 hpi and 4 dpi for M. marinum. Triplicate samples for each infection condition were compared with samples from control embryos (injected with PBS) using a common reference microarray design. The raw data were deposited in the Gene Expression Omnibus database under accession number GSE35474. The data derived from embryonic infections were compared with data from a study in which adult zebrafish were infected intraperitoneally with M. marinum Mma20, after which RNA samples were taken at 1 dpi and 6 dpi [22]. The dose of the Mma20 strain used in the adult infection study was lethal within days after the final sampling point at 6 dpi. Only genes relevant to this paper were included in the heatmap. All selected genes are represented by a minimum of two probes that showed significant up or downregulation (significance cut-offs for the ratios of infected versus control groups were set at 2 -fold with $P<10^{-5}$ ). Upregulation is indicated by increasingly bright shades of yellow, and downregulation is indicated by increasingly bright shades of blue. It should be noted that the genes listed in this figure are named according to sequence homology with mammalian counterparts and in most cases have not yet been confirmed functionally. 
show an immune response, although it was still weaker than the response to E. tarda or $S$. typhimurium infection at $8 \mathrm{hpi}$. In adults, the immune response to $M$. marinum infection has been shown to develop in a similar manner, with hardly any induction of proinflammatory genes at $1 \mathrm{dpi}$ and a stronger response at $6 \mathrm{dpi}$, when the fish began to show symptoms of disease [22]. Infections with E. tarda and S. typhimurium resulted in a remarkably similar transcriptome. Nevertheless, subtle differences were observed, like the upregulation of Tlr3 that was specific to $E$. tarda infection in this data set, and the variation in the panel of cytokines expressed upon these infections.

Interestingly, various PRRs, for example, Tlr5a and 5b, showed increased expression upon infection, most likely indicating an elevated state of awareness needed to identify the invading pathogens. In contrast, the fish-specific Tlr18, the scavenger receptors CD36, scarb1, and scarb2, and the C-type lectin $\mathrm{Mbl}$ were downregulated in some conditions. In many cases, signaling intermediates downstream of PRRs were upregulated, relaying and possibly amplifying the activating signals they receive from their respective receptors. A wide range of transcription factors with well-established functions in immunity (e.g., Atf3, Jun and Fos, Rel, and the IRF and Stat family members) were significantly upregulated under all conditions tested, except for the 8 hpi time point of $M$. marinum infection, whereas we observed upregulation of transcription factors of the oncogenic Myc family mainly in adult fish. The hematopoietic transcription factor Spil (Pu.1) was upregulated in M. marinum infection of embryos and adults. Genes for the key pro-inflammatory cytokines, like TNF $\alpha$ (two genes in zebrafish: tnfa and tnfb), IL1 $\beta$, and IL8, and for the anti-inflammatory cytokine IL10 were induced by infection with any of the three pathogens. Other cytokines appeared to be more specific for certain pathogens or might not be expressed at the specific time point of infection that we sampled.

We also observed increased expression of genes involved in effector mechanisms. However, upregulation of the genes encoding lysozyme, myeloperoxidase, and iNos was detectable only in adult zebrafish infected with M. marinum. Infection with any of the three pathogens led to increased gene expression of $n c f 1$, a subunit of the neutrophil NADPH oxidase complex. Proteases are an important part of the innate immune response, functioning in reorganizing the extracellular matrix to allow leukocyte migration, in degradation of microbes, and in processing of cytokines. In adult zebrafish infected with $M$. marinum, we observed upregulation of cathepsin-like $1 \mathrm{a}$ and $1 \mathrm{~b}$ (ctsl1a and ctsl1b), members of lysosomal cathepsin family that aids in the destruction of microbes. Expression levels of casp6 and caspb, members of the cysteine-aspartic acid protease (caspase) family involved in apoptosis, were downregulated at different stages of infection in adults and embryos. The matrix metalloproteinase (mmp) genes 9 and $m m p 13$ proved to be excellent markers for infection, since their gene expression was induced by E. tarda, S. typhimurium and M. marinum.

Our data further suggest that complement activation plays an important role during the early innate immune response, since a large number of complement factor genes show increased expression upon infection. Upregulated expression of the autophagy marker genes $l c 3$ and gabarap in adults infected with $M$. marinum hints towards a role for autophagy in the control of this infection. Intriguingly, a macrophage-expressed gene with unknown function in immunity, mpeg1 [87], is downregulated during the embryonic immune response against all three pathogens. The mouse homolog of this gene encodes a perforin-like protein that is expressed in mature macrophages and prion-infected brain cells [189]. We have also observed specific upregulation of genes with as of yet unknown function in immunity, like immunoresponsive gene 1 (irg1). This gene is highly conserved in vertebrates and has high homology to bacterial methylcitrate dehydrogenase [190]. We also included some genes involved in adaptive immunity in our comparison, the lymphocyte marker ragl, the immunoglobulin heavy chain gene ighm, and the antigen-presenting major histocompatibility complex class I UEA gene (mhcluea). Even though no cells of the adaptive immune system are present yet, embryos infected with E. tarda or S. typhimurium increase the expression of the MHC I gene. Finally, upon infection with S. typhimurium and M. marimum, we observe up and downregulation of chitinases, a family of genes which has been attributed a role during the host-microbial interactions involved in the development of acute and chronic inflammatory conditions [191].

\section{Discussion}

Zebrafish infectious disease models have started to make an important contribution to the understanding of hostpathogen interaction mechanisms. A good example is the discovery of the mechanism whereby a mycobacterial virulence factor (ESAT6) induces mmp9 expression in host epithelial cells neighboring infected macrophages, which enhances macrophage recruitment and formation of granuloma-like aggregates that provide a replication niche for mycobacteria [2]. The combination of genetics and in vivo imaging in zebrafish embryos is unparalleled in other vertebrate models. Furthermore, zebrafish embryos provide an ideal model for high-throughput in vivo screening of antimicrobial drug candidates or novel vaccine candidates $[192,193]$. Knowledge of the zebrafish immune system is also important in high-throughput screening for cancer in zebrafish embryos [194]. However, many aspects of zebrafish immunity still require further characterization and validation.

Currently available transgenic lines clearly distinguish macrophages (marked by csflr/fms and mpeg1) from neutrophils (marked by $m p x$ and lyz) in embryos and larvae, but there is insufficient knowledge of surface markers to identify different macrophage and neutrophil subpopulations. Similar to mammals, there is evidence of the existence of subpopulations of classically activated macrophages (M1: high producers of proinflammatory mediators, ROS, and NO) and alternatively activated macrophages (M2: high producers of anti-inflammatory mediators) in fish [195]. The polarization of macrophages towards these subtypes plays 
a critical role in the pathology of both infectious diseases and cancer [196]. Furthermore, different subpopulations of mammalian neutrophils (N1 and N2) have been recently described that display pro- and antitumorigenic properties [197] and that probably will also turn out to have distinctive functions during infectious disease pathology. Tumor implants in zebrafish embryos were shown to attract a heterogeneous population of leukocytes, including cells that express arginase, a marker of alternatively activated macrophages [177]. In addition, the neutrophil markers $m p x$, $m y c h$, and $l y z$ do not show complete overlap [177, 198], and markers such as cxcr3.2 and ptpn6, which are macrophage specific in one-day-old embryos, also label a subset of neutrophils at later stages [87]. Future development of transgenic lines that can distinguish these multiple myeloid subsets would further strengthen the use of zebrafish models for innate immunity and infectious disease studies.

As detailed in this paper, counterparts of the major vertebrate PRRs and downstream signaling components have been identified in zebrafish, but relatively few have thus far been functionally studied in infectious disease models. Recently, new PRRs have been described in mammals, like the INF-inducible dsRNA-activated protein kinase R (PKR) [199], the cytosolic DNA sensor DNA-dependent activator of IFN-regulatory factors (DAI) [200], and a cytosolic DNA receptor named AIM2 (absent in melanoma 2) [201]. Thus far, only the zebrafish homolog for PKR has been identified. Furthermore, autophagic adaptors known as sequestosome 1/p62-like receptors (SLRs), conserved between zebrafish and human, have recently been suggested as a new category of PRRs, since they have the ability to recognize and capture targets for immune-related autophagy [202].

Various datasets derived from transcriptome analyses have shown the specificity of immune responses to different pathogens. In future studies, the analysis of these responses can be refined by FACS sorting of immune cell populations from infected embryos, using labeled pathogens in combination with transgenic lines for different immune cell types. For example, it now comes within reach to aim at dissecting the differences in gene expression between M. marinum-infected macrophages inside a granuloma and recently attracted uninfected macrophages. In addition, simultaneous profiling of pathogen and host genes will be a challenging approach to help unravel the complex mechanisms underlying hostpathogen interactions. Transcriptome analysis only reveals altered RNA levels upon infection, and therefore, the application of proteomic and epigenetic analyses are needed to study the regulation of immune responses on different levels. Transcriptome studies have revealed infection responsiveness of many genes that have not yet been well studied (for example, dram 1, mpeg1, irgl, and irgll, mentioned above) and an emerging immune function for several chitinaselike proteins during infection [13, 37, 123]. Many zebrafish infection models have been described here and in other recent papers $[4,203,204]$ that can be used to investigate the functions of these genes in different pathogenic interactions, either using morpholino knockdown in embryos or using stable knockout lines which nowadays can be identified very efficiently by high-throughput resequencing of mutant libraries or by targeted knock-down approaches using technologies such as zinc finger nucleases (ZFNs) or transcription activator-like effector nucleases (TALENs) [205].

\section{Acknowledgments}

The authors thank Dan Klionsky (University of Michigan) for the GFP-Lc3 zebrafish line, Maria Forlenza (Wageningen University) for the antinitrotyrosine antibody, and Phil Elks for critically reading the paper. Infectious disease research in our laboratory is supported by the Smart Mix Program of the Netherlands Ministry of Economic Affairs and the Ministry of Education, Culture and Science, the European Commission 7th framework project ZF-HEALTH (HEALTH-F4-2010-242048), and the European Marie-Curie Initial Training Network FishForPharma (PITN-GA-2011289209).

\section{References}

[1] D. M. Tobin, J. C. Vary, J. P. Ray et al., "The lta4h locus modulates susceptibility to mycobacterial infection in zebrafish and humans," Cell, vol. 140, no. 5, pp. 717-730, 2010.

[2] H. E. Volkman, T. C. Pozos, J. Zheng, J. M. Davis, J. F. Rawls, and L. Ramakrishnan, "Tuberculous granuloma induction via interaction of a bacterial secreted protein with host epithelium," Science, vol. 327, no. 5964, pp. 466-469, 2010.

[3] M. Ludwig, N. Palha, C. Torhy et al., "Whole-body analysis of a viral infection: vascular endothelium is a primary target of Infectious hematopoietic necrosis virus in zebrafish larvae," PLoS Pathogens, vol. 7, no. 2, Article ID e1001269, 2011.

[4] A. H. Meijer and H. P. Spaink, "Host-Pathogen interactions made transparent with the zebrafish model," Current Drug Targets, vol. 12, no. 7, pp. 1000-1017, 2011.

[5] C. Sullivan and C. H. Kim, "Zebrafish as a model for infectious disease and immune function," Fish and Shellfish Immunology, vol. 25, no. 4, pp. 341-350, 2008.

[6] J. P. Allen and M. N. Neely, "Trolling for the ideal model host: zebrafish take the bait," Future Microbiology, vol. 5, no. 4, pp. 563-569, 2010.

[7] J. R. Mathias, K. B. Walters, and A. Huttenlocher, "Neutrophil motility in vivo using zebrafish.," Methods in Molecular Biology, vol. 571, pp. 151-166, 2009.

[8] S. A. Renshaw, C. A. Loynes, D. M. I. Trushell, S. Elworthy, P. W. Ingham, and M. K. B. Whyte, "Atransgenic zebrafish model of neutrophilic inflammation," Blood, vol. 108, no. 13, pp. 3976-3978, 2006.

[9] C. Hall, M. Flores, T. Storm, K. Crosier, and P. Crosier, "The zebrafish lysozyme C promoter drives myeloid-specific expression in transgenic fish," BMC Developmental Biology, vol. 7, article no. 42, 2007.

[10] C. Gray, C. A. Loynes, M. K. B. Whyte, D. C. Crossman, S. A. Renshaw, and T. J. A. Chico, "Simultaneous intravital imaging of macrophage and neutrophil behaviour during inflammation using a novel transgenic zebrafish," Thrombosis and Haemostasis, vol. 105, no. 5, pp. 811-819, 2011.

[11] F. Ellett, L. Pase, J. W. Hayman, A. Andrianopoulos, and G. J. Lieschke, "mpeg1 promoter transgenes direct macrophagelineage expression in zebrafish," Blood, vol. 117, no. 4, pp. e49-e56, 2011. 
[12] P. Herbomel, B. Thisse, and C. Thisse, "Ontogeny and behaviour of early macrophages in the zebrafish embryo," $D e$ velopment, vol. 126, no. 17, pp. 3735-3745, 1999.

[13] O. W. Stockhammer, A. Zakrzewska, Z. Hegedûs, H. P. Spaink, and A. H. Meijer, "Transcriptome profiling and functional analyses of the zebrafish embryonic innate immune response to Salmonella infection," Journal of Immunology, vol. 182, no. 9, pp. 5641-5653, 2009.

[14] S. H. Lam, H. L. Chua, Z. Gong, T. J. Lam, and Y. M. Sin, "Development and maturation of the immune system in zebrafish, Danio rerio: a gene expression profiling, in situ hybridization and immunological study," Developmental and Comparative Immunology, vol. 28, no. 1, pp. 9-28, 2004.

[15] R. Medzhitov and C. Janeway Jr., "Innate immune recognition: mechanisms and pathways," Immunological Reviews, vol. 173, pp. 89-97, 2000.

[16] A. A. Beg, "Endogenous ligands of Toll-like receptors: implications for regulating inflammatory and immune responses," Trends in Immunology, vol. 23, no. 11, pp. 509-512, 2002.

[17] P. Matzinger, "An innate sense of danger," Annals of the New York Academy of Sciences, vol. 961, pp. 341-342, 2002.

[18] T. H. Mogensen, "Pathogen recognition and inflammatory signaling in innate immune defenses," Clinical Microbiology Reviews, vol. 22, no. 2, pp. 240-273, 2009.

[19] S. Akira and K. Takeda, "Toll-like receptor signalling," Nature Reviews Immunology, vol. 4, no. 7, pp. 499-511, 2004.

[20] S. Akira, S. Uematsu, and O. Takeuchi, "Pathogen recognition and innate immunity," Cell, vol. 124, no. 4, pp. 783-801, 2006.

[21] M. Forlenza, J. P. Scharsack, N. M. Kachamakova, A. J. Taverne-Thiele, J. H. W. M. Rombout, and G. F. Wiegertjes, "Differential contribution of neutrophilic granulocytes and macrophages to nitrosative stress in a host-parasite animal model," Molecular Immunology, vol. 45, no. 11, pp. 31783189, 2008.

[22] A. M. van der Sar, H. P. Spaink, A. Zakrzewska, W. Bitter, and A. H. Meijer, "Specificity of the zebrafish host transcriptome response to acute and chronic mycobacterial infection and the role of innate and adaptive immune components," Molecular Immunology, vol. 46, no. 11-12, pp. 2317-2332, 2009.

[23] B. Lemaitre, E. Nicolas, L. Michaut, J. M. Reichhart, and J. A. Hoffmann, "The dorsoventral regulatory gene cassette spatzle/Toll/Cactus controls the potent antifungal response in Drosophila adults," Cell, vol. 86, no. 6, pp. 973-983, 1996.

[24] L. A. J. O'Neill and A. G. Bowie, "The family of five: TIRdomain-containing adaptors in Toll-like receptor signalling," Nature Reviews Immunology, vol. 7, no. 5, pp. 353-364, 2007.

[25] M. Miettinen, T. Sareneva, I. Julkunen, and S. Matikainen, "IFNs activate toll-like receptor gene expression in viral infections," Genes and Immunity, vol. 2, no. 6, pp. 349-355, 2001.

[26] K. Takeda and S. Akira, "Microbial recognition by Toll-like receptors," Journal of Dermatological Science, vol. 34, no. 2, pp. 73-82, 2004.

[27] C. Jault, L. Pichon, and J. Chluba, "Toll-like receptor gene family and TIR-domain adapters in Danio rerio," Molecular Immunology, vol. 40, no. 11, pp. 759-771, 2004.

[28] A. H. Meijer, S. F. Gabby Krens, I. A. Medina Rodriguez et al., "Expression analysis of the Toll-like receptor and TIR domain adaptor families of zebrafish," Molecular Immunology, vol. 40, no. 11, pp. 773-783, 2004.
[29] Y. Palti, "Toll-like receptors in bony fish: from genomics to function," Developmental and Comparative Immunology, vol. 35, no. 12, pp. 1263-1272, 2011.

[30] C. M. S. Ribeiro, T. Hermsen, A. J. Taverne-Thiele, H. F. J. Savelkoul, and G. F. Wiegertjes, "Evolution of recognition of ligands from gram-positive bacteria: similarities and differences in the TLR2-mediated response between mammalian vertebrates and teleost fish," Journal of Immunology, vol. 184, no. 5, pp. 2355-2368, 2010.

[31] A. Matsuo, H. Oshiumi, T. Tsujita et al., "Teleost TLR22 recognizes RNA duplex to induce IFN and protect cells from birnaviruses," Journal of Immunology, vol. 181, no. 5, pp. 3474-3485, 2008.

[32] M. P. Sepulcre, F. Alcaraz-Pérez, A. López-Muñoz et al., "Evolution of lipopolysaccharide (LPS) recognition and signaling: fish TLR4 does not recognize LPS and negatively regulates NF- $\kappa$ B activation," Journal of Immunology, vol. 182, no. 4, pp. 1836-1845, 2009.

[33] C. Sullivan, J. Charette, J. Catchen et al., "The gene history of zebrafish tlr4a and tlr $4 \mathrm{~b}$ is predictive of their divergent functions," Journal of Immunology, vol. 183, no. 9, pp. 58965908, 2009.

[34] C. Stein, M. Caccamo, G. Laird, and M. Leptin, "Conservation and divergence of gene families encoding components of innate immune response systems in zebrafish," Genome Biology, vol. 8, no. 11, article no. R251, 2007.

[35] J. M. Bates, J. Akerlund, E. Mittge, and K. Guillemin, "Intestinal alkaline phosphatase detoxifies lipopolysaccharide and prevents inflammation in zebrafish in response to the gut microbiota," Cell Host and Microbe, vol. 2, no. 6, pp. 371-382, 2007.

[36] A. M. Van Der Sar, O. W. Stockhammer, C. Van Der Laan, H. P. Spaink, W. Bitter, and A. H. Meijer, "MyD88 innate immune function in a zebrafish embryo infection model," Infection and Immunity, vol. 74, no. 4, pp. 2436-2441, 2006.

[37] O. W. Stockhammer, H. Rauwerda, F. R. Wittink, T. M. Breit, A. H. Meijer, and H. P. Spaink, "Transcriptome analysis of Traf6 function in the innate immune response of zebrafish embryos," Molecular Immunology, vol. 48, no. 1-3, pp. 179190, 2010.

[38] A. Ordas, Z. Hegedus, C. V. Henkel et al., "Deep sequencing of the innate immune transcriptomic response of zebrafish embryos to Salmonella infection," Fish and Shellfish Immunology, vol. 31, no. 5, pp. 716-724, 2011.

[39] K. Kersse, M. J. M. Bertrand, M. Lamkanfi, and P. Vandenabeele, "NOD-like receptors and the innate immune system: coping with danger, damage and death," Cytokine and Growth Factor Reviews, vol. 22, no. 5-6, pp. 257-276, 2011.

[40] T. D. Kanneganti, M. Lamkanfi, and G. Núñez, "Intracellular NOD-like receptors in host defense and disease," Immunity, vol. 27, no. 4, pp. 549-559, 2007.

[41] S. E. Girardin, I. G. Boneca, L. A. M. Carneiro et al., "Nod1 detects a unique muropeptide from gram-negative bacterial peptidoglycan," Science, vol. 300, no. 5625, pp. 1584-1587, 2003.

[42] S. E. Girardin, J. P. Hugot, and P. J. Sansonetti, "Lessons from Nod2 studies: towards a link between Crohn's disease and bacterial sensing," Trends in Immunology, vol. 24, no. 12, pp. 652-658, 2003.

[43] G. K. Silva, F. R. S. Gutierrez, P. M. M. Guedes et al., "Cutting edge: nucleotide-binding oligomerization domain 1dependent responses account for murine resistance against Trypanosoma cruzi infection," Journal of Immunology, vol. 184, no. 3, pp. 1148-1152, 2010. 
[44] M. H. Shaw, T. Reimer, C. Sánchez-Valdepeñas et al., "T cell-intrinsic role of Nod2 in promoting type 1 immunity to Toxoplasma gondii," Nature Immunology, vol. 10, no. 12, pp. 1267-1274, 2009.

[45] N. Inohara, T. Koseki, J. Lin et al., "An induced proximity model for NF- $\kappa \mathrm{B}$ activation in the Nod1/RICK and RIP signaling pathways," Journal of Biological Chemistry, vol. 275, no. 36, pp. 27823-27831, 2000.

[46] K. Kobayashi, N. Inohara, L. D. Hernandez et al., "RICK/ Rip2/CARDIAK mediates signalling for receptors of the innate and adaptive immune systems," Nature, vol. 416, no. 6877, pp. 194-199, 2002.

[47] J. H. Park, Y. G. Kim, M. Shaw et al., "Nod1/RICK and TLR signaling regulate chemokine and antimicrobial innate immune responses in mesothelial cells," Journal of Immunology, vol. 179, no. 1, pp. 514-521, 2007.

[48] E. Voss, J. Wehkamp, K. Wehkamp, E. F. Stange, J. M. Schröder, and J. Harder, "NOD2/CARD15 mediates induction of the antimicrobial peptide human beta-defensin-2," Journal of Biological Chemistry, vol. 281, no. 4, pp. 20052011, 2006.

[49] E. Noguchi, Y. Homma, X. Kang, M. G. Netea, and X. Ma, "A Crohn's disease-associated NOD2 mutation suppresses transcription of human IL10 by inhibiting activity of the nuclear ribonucleoprotein hnRNP-A1," Nature Immunology, vol. 10, no. 5, pp. 471-479, 2009.

[50] F. Martinon, K. Burns, and J. Tschopp, “The Inflammasome: a molecular platform triggering activation of inflammatory caspases and processing of proIL- $\beta$," Molecular Cell, vol. 10, no. 2, pp. 417-426, 2002.

[51] K. Tsuchiya, H. Hara, I. Kawamura et al., "Involvement of absent in melanoma 2 in inflammasome activation in macrophages infected with Listeria monocytogenes," Journal of Immunology, vol. 185, no. 2, pp. 1186-1195, 2010.

[52] D. L. Kastner, I. Aksentijevich, and R. Goldbach-Mansky, "Autoinflammatory disease reloaded: a clinical perspective," Cell, vol. 140, no. 6, pp. 784-790, 2010.

[53] S. C. Eisenbarth, O. R. Colegio, W. O'Connor, F. S. Sutterwala, and R. A. Flavell, "Crucial role for the Nalp3 inflammasome in the immunostimulatory properties of aluminium adjuvants," Nature, vol. 453, no. 7198, pp. 1122 1126, 2008.

[54] M. Chamaillard, S. E. Girardin, J. Viala, and D. J. Philpott, "Nods, nalps and naip: intracellular regulators of bacterialinduced inflammation," Cellular Microbiology, vol. 5, no. 9, pp. 581-592, 2003.

[55] T. Henry, A. Brotcke, D. S. Weiss, L. J. Thompson, and D. M. Monack, "Type I interferon signaling is required for activation of the inflammasome during Francisella infection," Journal of Experimental Medicine, vol. 204, no. 5, pp. 987994, 2007.

[56] K. J. Laing, M. K. Purcell, J. R. Winton, and J. D. Hansen, "A genomic view of the NOD-like receptor family in teleost fish: identification of a novel NLR subfamily in zebrafish," BMC Evolutionary Biology, vol. 8, no. 1, article no. 42, 2008.

[57] S. H. Oehlers, M. V. Flores, C. J. Hall, S. Swift, K. E. Crosier, and P. S. Crosier, "The inflammatory bowel disease (IBD) susceptibility genes NOD1 and NOD2 have conserved antibacterial roles in zebrafish," Disease Models and Mechanisms, vol. 4, no. 6, pp. 832-841, 2011.

[58] Y. M. Loo and M. Gale, "Immune signaling by RIG-I-like receptors," Immunity, vol. 34, no. 5, pp. 680-692, 2011.

[59] D. C. Kang, R. V. Gopalkrishnan, L. Lin et al., "Expression analysis and genomic characterization of human melanoma differentiation associated gene-5, mda-5: a novel type I interferon-responsive apoptosis-inducing gene," Oncogene, vol. 23, no. 9, pp. 1789-1800, 2004.

[60] M. Yoneyama, M. Kikuchi, T. Natsukawa et al., "The RNA helicase RIG-I has an essential function in double-stranded RNA-induced innate antiviral responses," Nature Immunology, vol. 5, no. 7, pp. 730-737, 2004.

[61] T. Saito, R. Hirai, Y. M. Loo et al., "Regulation of innate antiviral defenses through a shared repressor domain in RIG1 and LGP2," Proceedings of the National Academy of Sciences of the United States of America, vol. 104, no. 2, pp. 582-587, 2007.

[62] M. Yoneyama, M. Kikuchi, K. Matsumoto et al., "Shared and unique functions of the $\mathrm{DExD} / \mathrm{H}$-box helicases RIG-I, MDA5, and LGP2 in antiviral innate immunity," Journal of Immunology, vol. 175, no. 5, pp. 2851-2858, 2005.

[63] I. Scott, "The role of mitochondria in the mammalian antiviral defense system," Mitochondrion, vol. 10, no. 4, pp. 316-320, 2010.

[64] S. Paz, Q. Sun, P. Nakhaei et al., "Induction of IRF-3 and IRF-7 phosphorylation following activation of the RIG-I pathway," Cellular and Molecular Biology, vol. 52, no. 1, pp. 17-28, 2006.

[65] Y. M. Loo, J. Fornek, N. Crochet et al., "Distinct RIG-I and MDA5 signaling by RNA viruses in innate immunity," Journal of Virology, vol. 82, no. 1, pp. 335-345, 2008.

[66] J. Zou, M. Chang, P. Nie, and C. J. Secombes, "Origin and evolution of the RIG-I like RNA helicase gene family," $B M C$ Evolutionary Biology, vol. 9, no. 1, article no. 85, 2009.

[67] D. Aggad, M. Mazel, P. Boudinot et al., "The two groups of zebrafish virus-induced interferons signal via distinct receptors with specific and shared chains," Journal of Immunology, vol. 183, no. 6, pp. 3924-3931, 2009.

[68] S. Biacchesi, M. LeBerre, A. Lamoureux et al., "Mitochondrial antiviral signaling protein plays a major role in induction of the fish innate immune response against RNA and DNA viruses," Journal of Virology, vol. 83, no. 16, pp. 78157827, 2009.

[69] F. Sun, Y.-B. Zhang, T.-K. Liu, J. Shi, B. Wang, and J.F. Gui, "Fish MITA serves as a mediator for distinct fish IFN gene activation dependent on IRF3 or IRF7," Journal of Immunology, vol. 187, no. 5, pp. 2531-2539, 2011.

[70] N. Medic, F. Vita, R. Abbate et al., "Mast cell activation by myelin through scavenger receptor," Journal of Neuroimmunology, vol. 200, no. 1-2, pp. 27-40, 2008.

[71] J. E. Murphy, P. R. Tedbury, S. Homer-Vanniasinkam, J. H. Walker, and S. Ponnambalam, "Biochemistry and cell biology of mammalian scavenger receptors," Atherosclerosis, vol. 182, no. 1, pp. 1-15, 2005.

[72] A. Plüddemann, C. Neyen, and S. Gordon, "Macrophage scavenger receptors and host-derived ligands," Methods, vol. 43, no. 3, pp. 207-217, 2007.

[73] T. Areschoug and S. Gordon, "Pattern recognition receptors and their role in innate immunity: focus on microbial protein ligands," Contributions to Microbiology, vol. 15, pp. 45-60, 2008.

[74] S. E. Doyle, R. M. O’Connell, G. A. Miranda et al., "Toll-like receptors induce a phagocytic gene program through $\mathrm{p} 38$," Journal of Experimental Medicine, vol. 199, no. 1, pp. 81-90, 2004.

[75] K. Hoebe, P. Georgel, S. Rutschmann et al., "CD36 is a sensor of diacylglycerides," Nature, vol. 433, no. 7025, pp. 523-527, 2005. 
[76] P. Jeannin, B. Bottazzi, M. Sironi et al., "Complexity and complementarity of outer membrane protein A recognition by cellular and humoral innate immunity receptors," Immunity, vol. 22, no. 5, pp. 551-560, 2005.

[77] L. Peiser, P. J. Gough, T. Kodama, and S. Gordon, "Macrophage class A scavenger receptor-mediated phagocytosis of Escherichia coli: role of cell heterogeneity, microbial strain, and culture conditions in vitro," Infection and Immunity, vol. 68, no. 4, pp. 1953-1963, 2000.

[78] L. Peiser, M. P. J. De Winther, K. Makepeace et al., "The class A macrophage scavenger receptor is a major pattern recognition receptor for Neisseria meningitidis which is independent of lipopolysaccharide and not required for secretory responses," Infection and Immunity, vol. 70, no. 10, pp. 5346-5354, 2002.

[79] M. S. Arredouani, Z. Yang, A. Imrich, Y. Ning, G. Qin, and L. Kobzik, "The macrophage scavenger receptor SR-AI/II and lung defense against pneumococci and particles," American Journal of Respiratory Cell and Molecular Biology, vol. 35, no. 4, pp. 474-478, 2006.

[80] O. Elomaa, M. Kangas, C. Sahlberg et al., "Cloning of a novel bacteria-binding receptor structurally related to scavenger receptors and expressed in a subset of macrophages," Cell, vol. 80, no. 4, pp. 603-609, 1995.

[81] M. Arredouani, Z. Yang, Y. Y. Ning et al., "The scavenger receptor MARCO is required for lung defense against pneumococcal pneumonia and inhaled particles," Journal of Experimental Medicine, vol. 200, no. 2, pp. 267-272, 2004.

[82] S. Mukhopadhyay, Y. Chen, M. Sankala et al., "MARCO, an innate activation marker of macrophages, is a class A scavenger receptor for Neisseria meningitidis," European Journal of Immunology, vol. 36, no. 4, pp. 940-949, 2006.

[83] D. M. E. Bowdish, K. Sakamoto, M. J. Kim et al., "MARCO, TLR2, and CD14 are required for macrophage cytokine responses to mycobacterial trehalose dimycolate and $\mathrm{Myco}$ bacterium tuberculosis," PLoS Pathogens, vol. 5, no. 6, Article ID e1000474, 2009.

[84] L. M. Stuart, J. Deng, J. M. Silver et al., "Response to Staphylococcus aureus requires CD36-mediated phagocytosis triggered by the $\mathrm{COOH}$-terminal cytoplasmic domain," Journal of Cell Biology, vol. 170, no. 3, pp. 477-485, 2005.

[85] T. G. Vishnyakova, R. Kurlander, A. V. Bocharov et al., "CLA1 and its splicing variant CLA-2 mediate bacterial adhesion and cytosolic bacterial invasion in mammalian cells," Proceedings of the National Academy of Sciences of the United States of America, vol. 103, no. 45, pp. 16888-16893, 2006.

[86] V. Wittamer, J. Y. Bertrand, P. W. Gutschow, and D. Traver, "Characterization of the mononuclear phagocyte system in zebrafish,” Blood, vol. 117, no. 26, pp. 7126-7135, 2011.

[87] A. Zakrzewska, C. Cui, O. W. Stockhammer, E. L. Benard, H. P. Spaink, and A. H. Meijer, "Macrophage-specific gene functions in Spil-directed innate immunity," Blood, vol. 116, no. 3, pp. e1-e11, 2010.

[88] P. Encinas, M. A. Rodriguez-Milla, B. Novoa, A. Estepa, A. Figueras, and J. Coll, "Zebrafish fin immune responses during high mortality infections with viral haemorrhagic septicemia rhabdovirus. A proteomic and transcriptomic approach," BMC Genomics, vol. 11, no. 1, article no. 518, 2010.

[89] A. N. Zelensky and J. E. Gready, "The C-type lectin-like domain superfamily," FEBS Journal, vol. 272, no. 24, pp. 6179-6217, 2005.
[90] M. J. Robinson, D. Sancho, E. C. Slack, S. LeibundGutLandmann, and C. R. Sousa, "Myeloid C-type lectins ininnate immunity," Nature Immunology, vol. 7, no. 12, pp. 1258 1265, 2006.

[91] L. L. Lanier, "NK cell recognition," Annual Review of Immunology, vol. 23, pp. 225-274, 2005.

[92] M. Takahashi, D. Iwaki, A. Matsushita et al., "Cloning and characterization of mannose-binding lectin from lamprey (Agnathans)," Journal of Immunology, vol. 176, no. 8, pp. 4861-4868, 2006.

[93] T. B. H. Geijtenbeek and S. I. Gringhuis, "Signalling through C-type lectin receptors: shaping immune responses," Nature Reviews Immunology, vol. 9, no. 7, pp. 465-479, 2009.

[94] J. Herre, A. S. J. Marshall, E. Caron et al., "Dectin-1 uses novel mechanisms for yeast phagocytosis in macrophages," Blood, vol. 104, no. 13, pp. 4038-4045, 2004.

[95] D. M. Underhill, E. Rossnagle, C. A. Lowell, and R. M. Simmons, "Dectin-1 activates Syk tyrosine kinase in a dynamic subset of macrophages for reactive oxygen production," Blood, vol. 106, no. 7, pp. 2543-2550, 2005.

[96] P. J. Tacken, R. Torensma, and C. G. Figdor, "Targeting antigens to dendritic cells in vivo," Immunobiology, vol. 211, no. 6-8, pp. 599-608, 2006.

[97] N. C. Rogers, E. C. Slack, A. D. Edwards et al., "Syk-dependent cytokine induction by dectin-1 reveals a novel pattern recognition pathway for C type lectins," Immunity, vol. 22, no. 4, pp. 507-517, 2005.

[98] K. Sato, X. L. Yang, T. Yudate et al., "Dectin-2 is a pattern recognition receptor for fungi that couples with the $\mathrm{Fc}$ receptor $\gamma$ chain to induce innate immune responses," Journal of Biological Chemistry, vol. 281, no. 50, pp. 38854-38866, 2006.

[99] S. Yamasaki, M. Matsumoto, O. Takeuchi et al., "C-type lectin Mincle is an activating receptor for pathogenic fungus, Malassezia," Proceedings of the National Academy of Sciences of the United States of America, vol. 106, no. 6, pp. 1897-1902, 2009.

[100] O. Gross, A. Gewies, K. Finger et al., "Card9 controls a nonTLR signalling pathway for innate anti-fungal immunity," Nature, vol. 442, no. 7103, pp. 651-656, 2006.

[101] S. I. Gringhuis, J. den Dunnen, M. Litjens, B. van het Hof, Y. van Kooyk, and T. H. Geijtenbeek, "C-Type Lectin DC-SIGN modulates toll-like receptor signaling via Raf-1 kinase-dependent acetylation of transcription factor NF- $\kappa \mathrm{B}$," Immunity, vol. 26, no. 5, pp. 605-616, 2007.

[102] A. N. Jackson, C. A. McLure, R. L. Dawkins, and P. J. Keating, "Mannose binding lectin (MBL) copy number polymorphism in Zebrafish (D. rerio) and identification of haplotypes resistant to L. anguillarum," Immunogenetics, vol. 59, no. 11, pp. 861-872, 2007.

[103] M. Fukuda, K. Ohtani, S.-J. Jang et al., "Molecular cloning and functional analysis of scavenger receptor zebrafish CLP1, Biochimica et Biophysica Acta, vol. 1810, no. 12, pp. 1150-1159, 2011.

[104] K. Ohtani, Y. Suzuki, S. Eda et al., "The membrane-type collectin CL-P1 is a scavenger receptor on vascular endothelial cells," Journal of Biological Chemistry, vol. 276, no. 47, pp. 44222-44228, 2001

[105] A. F. Lin, L. X. Xiang, Q. L. Wang, W. R. Dong, Y. F. Gong, and J. Z. Shao, "The DC-SIGN of zebrafish: insights into the existence of a CD209 homologue in a lower vertebrate and its involvement in adaptive immunity," Journal of Immunology, vol. 183, no. 11, pp. 7398-7410, 2009. 
[106] P. G. Panagos, K. P. Dobrinski, X. Chen et al., "Immunerelated, lectin-like receptors are differentially expressed in the myeloid and lymphoid lineages of zebrafish," Immunogenetics, vol. 58, no. 1, pp. 31-40, 2006.

[107] Z. Hegedus, A. Zakrzewska, V. C. Ágoston et al., "Deep sequencing of the zebrafish transcriptome response to mycobacterium infection," Molecular Immunology, vol. 46, no. 15, pp. 2918-2930, 2009.

[108] B. Lin, S. Chen, Z. Cao et al., "Acute phase response in zebrafish upon Aeromonas salmonicida and Staphylococcus aureus infection: striking similarities and obvious differences with mammals," Molecular Immunology, vol. 44, no. 4, pp. 295301, 2007.

[109] I. Rojo, O. M. de Ilárduya, A. Estonba, and M. A. Pardo, "Innate immune gene expression in individual zebrafish after Listonella anguillarum inoculation," Fish and Shellfish Immunology, vol. 23, no. 6, pp. 1285-1293, 2007.

[110] S. P. Commins, L. Borish, and J. W. Steinke, "Immunologic messenger molecules: cytokines, interferons, and chemokines," Journal of Allergy and Clinical Immunology, vol. 125, no. 2, supplement 2, pp. S53-S72, 2010.

[111] C. Perez, I. Albert, K. DeFay, N. Zachariades, L. Gooding, and M. Kriegler, "A nonsecretable cell surface mutant of tumor necrosis factor (TNF) kills by cell-to-cell contact," Cell, vol. 63, no. 2, pp. 251-258, 1990.

[112] D. P. Cerretti, C. J. Kozlosky, B. Mosley et al., "Molecular cloning of the interleukin-1 $\beta$ converting enzyme," Science, vol. 256, no. 5053, pp. 97-100, 1992.

[113] I. Lalani, K. Bhol, and A. R. Ahmed, "Interleukin-10: biology, role in inflammation and autoimmunity," Annals of Allergy, Asthma and Immunology, vol. 79, no. 6, pp. 469-484, 1997.

[114] H. Nomiyama, K. Hieshima, N. Osada et al., "Extensive expansion and diversification of the chemokine gene family in zebrafish: identification of a novel chemokine subfamily CX," BMC Genomics, vol. 9, article no. 222, 2008.

[115] M. O. Huising, R. J. M. Stet, H. F. J. Savelkoul, and B. M. L. Verburg-Van Kemenade, "The molecular evolution of the interleukin-1 family of cytokines; IL-18 in teleost fish," Developmental and Comparative Immunology, vol. 28, no. 5, pp. 395-413, 2004.

[116] D.-C. Zhang, Y.-Q. Shao, Y.-Q. Huang, and S.-G. Jiang, "Cloning, characterization and expression analysis of interleukin-10 from the zebrafish (Danio reriori)," Journal of Biochemistry and Molecular Biology, vol. 38, no. 5, pp. 571576, 2005.

[117] M. Varela, S. Dios, B. Novoa, and A. Figueras, "Characterisation, expression and ontogeny of interleukin-6 and its receptors in zebrafish (Danio rerio)," Developmental and Comparative Immunology, vol. 37, no. 1, pp. 97-106, 2012.

[118] L. Grayfer and M. Belosevic, "Molecular characterization of novel interferon gamma receptor 1 isoforms in zebrafish (Danio rerio) and goldfish (Carassius auratus L.)," Developmental \& Comparative Immunology, vol. 36, no. 2, pp. 408417, 2012.

[119] S. H. B. Oehlers, M. V. Flores, C. J. Hall et al., "Expression of zebrafish cxcl8 (interleukin-8) and its receptors during development and in response to immune stimulation," Developmental and Comparative Immunology, vol. 34, no. 3, pp. 352-359, 2010.

[120] L. M. van der Aa, M. Chadzinska, E. Tijhaar, P. Boudinot, and B. M. Lidy verburg-Van kemenade, "CXCL8 chemokines in teleost fish: two lineages with distinct expression profiles during early phases of inflammation," PLoS One, vol. 5, no. 8, Article ID e12384, 2010.
[121] A. E. Clatworthy, J. S. W. Lee, M. Leibman, Z. Kostun, A. J. Davidson, and D. T. Hung, "Pseudomonas aeruginosa infection of zebrafish involves both host and pathogen determinants," Infection and Immunity, vol. 77, no. 4, pp. 1293-1303, 2009.

[122] M. E. Pressley, P. E. Phelan, P. Eckhard Witten, M. T. Mellon, and C. H. Kim, "Pathogenesis and inflammatory response to Edwardsiella tardainfection in the zebrafish," Developmental and Comparative Immunology, vol. 29, no. 6, pp. 501-513, 2005.

[123] J. J. Van Soest, O. W. Stockhammer, A. Ordas, G. V. Bloemberg, H. P. Spaink, and A. H. Meijer, "Comparison of static immersion and intravenous injection systems for exposure of zebrafish embryos to the natural pathogen Edwardsiella tarda," BMC Immunology, vol. 12, Article ID 58, 2011.

[124] H. Clay, H. E. Volkman, and L. Ramakrishnan, "Tumor necrosis factor signaling mediates resistance to mycobacteria by inhibiting bacterial growth and macrophage death," Immunity, vol. 29, no. 2, pp. 283-294, 2008.

[125] F. J. Roca, I. Mulero, A. López-Muñoz et al., "Evolution of the inflammatory response in vertebrates: fish TNF- $\alpha$ is a powerful activator of endothelial cells but hardly activates phagocytes," Journal of Immunology, vol. 181, no. 7, pp. 50715081, 2008.

[126] D. Aggad, C. Stein, D. Sieger et al., "In vivo analysis of Ifn$\gamma 1$ and Ifn- $\gamma 2$ signaling in zebrafish," Journal of Immunology, vol. 185, no. 11, pp. 6774-6782, 2010.

[127] D. Sieger, C. Stein, D. Neifer, A. M. Van Der Sar, and M. Leptin, "The role of gamma interferon in innate immunity in the zebrafish embryo," Disease Models and Mechanisms, vol. 2, no. 11-12, pp. 571-581, 2009.

[128] O. J. Hamming, G. Lutfalla, J. P. Levraud, and R. Hartmann, "Crystal structure of zebrafish interferons I and II reveals conservation of type I interferon structure in vertebrates," Journal of Virology, vol. 85, no. 16, pp. 8181-8187, 2011.

[129] J. P. Levraud, P. Boudinot, I. Colin et al., "Identification of the zebrafish IFN receptor: implications for the origin of the vertebrate IFN system," Journal of Immunology, vol. 178, no. 7, pp. 4385-4394, 2007.

[130] K. Kwiatkowska and A. Sobota, "Signaling pathways in phagocytosis," BioEssays, vol. 21, no. 5, pp. 422-431, 1999.

[131] P. J. Sansonetti, “Phagocytosis, a cell biology view," Journal of Cell Science, vol. 113, part 19, pp. 3355-3356, 2000.

[132] A. Pitt, L. S. Mayorga, P. D. Stahl, and A. L. Schwartz, "Alterations in the protein composition of maturing phagosomes," Journal of Clinical Investigation, vol. 90, no. 5, pp. 1978-1983, 1992.

[133] T. E. Tjelle, T. Løvdal, and T. Berg, "Phagosome dynamics and function," BioEssays, vol. 22, no. 3, pp. 255-263, 2000.

[134] H. Tapper, "Out of the phagocyte or into its phagosome: signalling to secretion," European Journal of Haematology, vol. 57, no. 3, pp. 191-201, 1996.

[135] A. W. Segal, "How neutrophils kill microbes," Annual Review of Immunology, vol. 23, pp. 197-223, 2005.

[136] W. L. Lee, R. E. Harrison, and S. Grinstein, "Phagocytosis by neutrophils," Microbes and Infection, vol. 5, no. 14, pp. 12991306, 2003.

[137] S. Duclos and M. Desjardins, "Subversion of a young phagosome: the survival strategies of intracellular pathogens," Cellular Microbiology, vol. 2, no. 5, pp. 365-377, 2000.

[138] J. Gruenberg and F. G. Van Der Goot, "Mechanisms of pathogen entry through the endosomal compartments," 
Nature Reviews Molecular Cell Biology, vol. 7, no. 7, pp. 495504, 2006.

[139] J. M. Davis, H. Clay, J. L. Lewis, N. Ghori, P. Herbomel, and L. Ramakrishnan, "Real-time visualization of Mycobacteriummacrophage interactions leading to initiation of granuloma formation in zebrafish embryos," Immunity, vol. 17, no. 6, pp. 693-702, 2002.

[140] L. M. Stamm, J. H. Morisaki, L. Y. Gao et al., "Mycobacterium marinum escapes from phagosomes and is propelled by actin-based motility," Journal of Experimental Medicine, vol. 198, no. 9, pp. 1361-1368, 2003.

[141] N. van der Wel, D. Hava, D. Houben et al., "M. tuberculosis and $M$. leprae translocate from the phagolysosome to the cytosol in myeloid cells," Cell, vol. 129, no. 7, pp. 1287-1298, 2007.

[142] B. Levine, N. Mizushima, and H. W. Virgin, "Autophagy in immunity and inflammation," Nature, vol. 469, no. 7330, pp. 323-335, 2011.

[143] E. Itakura, C. Kishi, K. Inoue, and N. Mizushima, "Beclin 1 forms two distinct phosphatidylinositol 3-kinase complexes with mammalian Atg14 and UVRAG," Molecular Biology of the Cell, vol. 19, no. 12, pp. 5360-5372, 2008.

[144] H. Nakatogawa, J. Ishii, E. Asai, and Y. Ohsumi, "Atg4 recycles inappropriately lipidated Atg8 to promote autophagosome biogenesis," Autophagy, vol. 8, no. 2, 2012.

[145] M. A. Sanjuan and D. R. Green, "Eating for good health: linking autophagy and phagocytosis in host defense," Autophagy, vol. 4, no. 5, pp. 607-611, 2008.

[146] M. Ponpuak, A. S. Davis, E. A. Roberts et al., "Delivery of cytosolic components by autophagic adaptor protein p62 endows autophagosomes with unique antimicrobial properties," Immunity, vol. 32, no. 3, pp. 329-341, 2010.

[147] R. Sumpter and B. Levine, "Autophagy and innate immunity: triggering, targeting and tuning," Seminars in Cell and Developmental Biology, vol. 21, no. 7, pp. 699-711, 2010.

[148] C. Hall, M. V. Flores, A. Chien, A. Davidson, K. Crosier, and P. Crosier, "Transgenic zebrafish reporter lines reveal conserved Toll-like receptor signaling potential in embryonic myeloid leukocytes and adult immune cell lineages," Journal of Leukocyte Biology, vol. 85, no. 5, pp. 751-765, 2009.

[149] D. Le Guyader, M. J. Redd, E. Colucci-Guyon et al., "Origins and unconventional behavior of neutrophils in developing zebrafish," Blood, vol. 111, no. 1, pp. 132-141, 2008.

[150] E. Colucci-Guyon, J.-Y. Tinevez, S. A. Renshaw, and P. Herbomel, "Strategies of professional phagocytes in vivo: unlike macrophages, neutrophils engulf only surface-associated microbes," Journal of Cell Science, vol. 124, no. 18, pp. 30533059, 2011.

[151] J. Ulvila, L. M. Vanha-Aho, A. Kleino et al., "Cofilin regulator $14-3-3 \zeta$ is an evolutionarily conserved protein required for phagocytosis and microbial resistance," Journal of Leukocyte Biology, vol. 89, no. 5, pp. 649-659, 2011.

[152] C. He, C. R. Bartholomew, W. Zhou, and D. J. Klionsky, "Assaying autophagic activity in transgenic GFP-Lc3 and GFP-Gabarap zebrafish embryos," Autophagy, vol. 5, no. 4, pp. 520-526, 2009.

[153] A. Lu, X. Hu, J. Xue, J. Zhu, Y. Wang, and G. Zhou, "Gene expression profiling in the skin of zebrafish infected with Citrobacter freundii," Fish and Shellfish Immunology, vol. 32, no. 2, pp. 273-283, 2012.

[154] C. Delclaux, C. Delacourt, M. P. D'Ortho, V. Boyer, C. Lafuma, and A. Harf, "Role of gelatinase B and elastase in human polymorphonuclear neutrophil migration across basement membrane," American Journal of Respiratory Cell and Molecular Biology, vol. 14, no. 3, pp. 288-295, 1996.

[155] H. Sengelov, L. Kjeldsen, and N. Borregaard, "Control of exocytosis in early neutrophil activation," Journal of Immunology, vol. 150, no. 4, pp. 1535-1543, 1993.

[156] K. A. Joiner, T. Ganz, J. Albert, and D. Rotrosen, "The opsonizing ligand on Salmonella typhimurium influences incorporation of specific, but not azurophil, granule constituents into neutrophil phagosomes," Journal of Cell Biology, vol. 109, no. 6 I, pp. 2771-2782, 1989.

[157] M. Faurschou and N. Borregaard, "Neutrophil granules and secretory vesicles in inflammation," Microbes and Infection, vol. 5, no. 14, pp. 1317-1327, 2003.

[158] C. Zhang, J. Yang, J. D. Jacobs, and L. K. Jennings, "Interaction of myeloperoxidase with vascular NAD(P)H oxidasederived reactive oxygen species in vasculature: implications for vascular diseases," American Journal of Physiology, Heart and Circulatory Physiology, vol. 285, no. 6, pp. H2563-H2572, 2003.

[159] V. Brinkmann, U. Reichard, C. Goosmann et al., "Neutrophil extracellular traps kill bacteria," Science, vol. 303, no. 5663, pp. 1532-1535, 2004.

[160] J. D. Lambeth, "NOX enzymes and the biology of reactive oxygen," Nature Reviews Immunology, vol. 4, no. 3, pp. 181189, 2004.

[161] B. M. Babior, "The activity of leukocyte NADPH oxidase: regulation by p47PHOX cysteine and serine residues," Antioxidants and Redox Signaling, vol. 4, no. 1, pp. 35-38, 2002.

[162] D. Roos and C. C. Winterbourn, "Immunology: lethal weapons," Science, vol. 296, no. 5568, pp. 669-671, 2002.

[163] E. Peranzoni, I. Marigo, L. Dolcetti et al., "Role of arginine metabolism in immunity and immunopathology," Immunobiology, vol. 212, no. 9-10, pp. 795-812, 2008.

[164] C. Bogdan, "Nitric oxide and the immune response," Nature Immunology, vol. 2, no. 10, pp. 907-916, 2001.

[165] S. El-Gayar, H. Thüring-Nahler, J. Pfeilschifter, M. Röllinghoff, and C. Bogdan, "Translational control of inducible nitric oxide synthase by IL-13 and arginine availability in inflammatory macrophages," Journal of Immunology, vol. 171, no. 9, pp. 4561-4568, 2003.

[166] G. Wu and S. M. Morris, "Arginine metabolism: nitric oxide and beyond," Biochemical Journal, vol. 336, no. 1, pp. 1-17, 1998.

[167] S. Pfeiffer, A. Lass, K. Schmidt, and B. Mayer, "Protein tyrosine nitration in cytokine-activated murine macrophages. Involvement of a peroxidase/nitrite pathway rather than peroxynitrite," Journal of Biological Chemistry, vol. 276, no. 36, pp. 34051-34058, 2001.

[168] C. J. Hall, M. V. Flores, S. H. Oehlers et al., "Infectionresponsive expansion of the hematopoietic stem and progenitor cell compartment in zebrafish is dependent upon inducible nitric oxide," Cell Stem Cell, vol. 10, no. 2, pp. 198209, 2012.

[169] J. Arnhold and J. Flemmig, "Human myeloperoxidase in innate and acquired immunity," Archives of Biochemistry and Biophysics, vol. 500, no. 1, pp. 92-106, 2010.

[170] J. D. Shearer, J. R. Richards, C. D. Mills, and M. D. Caldwell, "Differential regulation of macrophage arginine metabolism: a proposed role in wound healing," American Journal of Physiology, Endocrinology and Metabolism, vol. 272, no. 2, pp. E181-E190, 1997.

[171] J. R. Mathias, B. J. Perrin, T. X. Liu, J. Kanki, A. T. Look, and A. Huttenlocher, "Resolution of inflammation by retrograde 
chemotaxis of neutrophils in transgenic zebrafish," Journal of Leukocyte Biology, vol. 80, no. 6, pp. 1281-1288, 2006.

[172] P. Niethammer, C. Grabher, A. T. Look, and T. J. Mitchison, "A tissue-scale gradient of hydrogen peroxide mediates rapid wound detection in zebrafish," Nature, vol. 459, no. 7249, pp. 996-999, 2009.

[173] Q. Deng, E. A. Harvie, and A. Huttenlocher, "Distinct signalling mechanisms mediate neutrophil attraction to bacterial infection and tissue injury," Cellular Microbiology, vol. 14, no. 4, pp. 517-528, 2012.

[174] S. K. Yoo, T. W. Starnes, Q. Deng, and A. Huttenlocher, "Lyn is a redox sensor that mediates leukocyte wound attraction in vivo," Nature, vol. 480, no. 7375, pp. 109-112, 2011.

[175] M. V. Flores, K. C. Crawford, L. M. Pullin, C. J. Hall, K. E. Crosier, and P. S. Crosier, "Dual oxidase in the intestinal epithelium of zebrafish larvae has anti-bacterial properties," Biochemical and Biophysical Research Communications, vol. 400, no. 1, pp. 164-168, 2010.

[176] K. M. Brothers, Z. R. Newman, and R. T. Wheeler, "Live imaging of disseminated candidiasis in zebrafish reveals role of phagocyte oxidase in limiting filamentous growth," Eukaryotic Cell, vol. 10, no. 7, pp. 932-944, 2011.

[177] Y. Feng, C. Santoriello, M. Mione, A. Hurlstone, and P. Martin, "Live imaging of innate immune cell sensing of transformed cells in zebrafish larvae: parallels between tumor initiation and wound inflammation," PLoS Biology, vol. 8, no. 12, Article ID e1000562, 2010.

[178] A. C. Hermann, P. J. Millard, S. L. Blake, and C. H. Kim, "Development of a respiratory burst assay using zebrafish kidneys and embryos," Journal of Immunological Methods, vol. 292, no. 1-2, pp. 119-129, 2004.

[179] S. Lepiller, V. Laurens, A. Bouchot, P. Herbomel, E. Solary, and J. Chluba, "Imaging of nitric oxide in a living vertebrate using a diaminofluorescein probe," Free Radical Biology and Medicine, vol. 43, no. 4, pp. 619-627, 2007.

[180] P. M. Elks, F. J. Van Eeden, G. Dixon et al., "Activation of hypoxia-inducible factor- $1 \alpha$ (hif- $1 \alpha$ ) delays inflammation resolution by reducing neutrophil apoptosis and reverse migration in a zebrafish inflammation model," Blood, vol. 118, no. 3, pp. 712-722, 2011.

[181] M. De Jong, H. Rauwerda, O. Bruning, J. Verkooijen, H. P. Spaink, and T. M. Breit, "RNA isolation method for single embryo transcriptome analysis in zebrafish," BMC Research Notes, vol. 3, article no. 73, 2010.

[182] A. H. Meijer, F. J. Verbeek, E. Salas-Vidal et al., "Transcriptome profiling of adult zebrafish at the late stage of chronic tuberculosis due to Mycobacterium marinum infection," Molecular Immunology, vol. 42, no. 10, pp. 1185-1203, 2005.

[183] Z. Wu, W. Zhang, Y. Lu, and C. Lu, "Transcriptome profiling of zebrafish infected with Streptococcus suis," Microbial Pathogenesis, vol. 48, no. 5, pp. 178-187, 2010.

[184] P. S. Srinivasa Rao, T. M. Lim, and K. Y. Leung, "Functional genomics approach to the identification of virulence genes involved in Edwardsiella tardapathogenesis," Infection and Immunity, vol. 71, no. 3, pp. 1343-1351, 2003.

[185] A. M. van der Sar, R. J. P. Musters, F. J. M. van Eeden, B. J. Appelmelk, C. M. J. E. Vandenbroucke-Grauls, and W. Bitter, "Zebrafish embryos as a model host for the real time analysis of Salmonella typhimurium infections," Cellular Microbiology, vol. 5, no. 9, pp. 601-611, 2003.

[186] D. M. Tobin and L. Ramakrishnan, "Comparative pathogenesis of Mycobacterium marinum and Mycobacterium tuberculosis," Cellular Microbiology, vol. 10, no. 5, pp. 1027 1039, 2008.
[187] H. E. Volkman, H. Clay, D. Beery, J. C. W. Chang, D. R. Sherman, and L. Ramakrishnan, "Tuberculous granuloma formation is enhanced by a Mycobacterium virulence determinant," PLoS Biology, vol. 2, no. 11, article e367, 2004.

[188] H. Clay, J. M. Davis, D. Beery, A. Huttenlocher, S. E. Lyons, and L. Ramakrishnan, "Dichotomous Role of the Macrophage in Early Mycobacterium marinum Infection of the Zebrafish," Cell Host and Microbe, vol. 2, no. 1, pp. 29-39, 2007.

[189] K. Spilsbury, M. A. O’Mara, Wan Man Wu, P. B. Rowe, G. Symonds, and Y. Takayama, "Isolation of a novel macrophage-specific gene by differential cDNA analysis," Blood, vol. 85, no. 6, pp. 1620-1629, 1995.

[190] B. Chen, D. Zhang, and J. W. Pollard, "Progesterone regulation of the mammalian ortholog of methylcitrate dehydratase (Immune Response Gene 1) in the uterine epithelium during implantation through the protein kinase C pathway," Molecular Endocrinology, vol. 17, no. 11, pp. 2340-2354, 2003.

[191] H. T. Tran, N. Barnich, and E. Mizoguchi, "Potential role of chitinases and chitin-binding proteins in host-microbial interactions during the development of intestinal inflammation," Histology and Histopathology, vol. 26, no. 11, pp. 14531464, 2011.

[192] R. Carvalho, J. de Sonneville, O. W. Stockhammer et al., "A high-throughput screen for tuberculosis progression," PLoS One, vol. 6, no. 2, Article ID e16779, 2011.

[193] E. J. M. Stoop, T. Schipper, S. K. Rosendahl Huber et al., "Zebrafish embryo screen for mycobacterial genes involved in the initiation of granuloma formation reveals a newly identified ESX-1 component," Disease Models and Mechanisms, vol. 4, no. 4, pp. 526-536, 2011.

[194] M. G. Morash, S. E. Douglas, A. Robotham, C. M. Ridley, J. W. Gallant, and K. H. Soanes, "The zebrafish embryo as a tool for screening and characterizing pleurocidin hostdefense peptides as anti-cancer agents," Disease Models and Mechanisms, vol. 4, no. 5, pp. 622-633, 2011.

[195] G. F. Wiegertjes and M. Forlenza, "Nitrosative stress during infection-induced inflammation in fish: lessons from a hostparasite infection model," Current Pharmaceutical Design, vol. 16, no. 38, pp. 4194-4202, 2010.

[196] F. O. Martinez, L. Helming, and S. Gordon, "Alternative activation of macrophages: an immunologic functional perspective," Annual Review of Immunology, vol. 27, pp. 451483, 2009.

[197] Z. G. Fridlender, J. Sun, S. Kim et al., "Polarization of tumorassociated neutrophil phenotype by TGF- $\beta$ : "N1" versus “N2" TAN," Cancer Cell, vol. 16, no. 3, pp. 183-194, 2009.

[198] A. H. Meijer, A. M. van der Sar, C. Cunha et al., "Identification and real-time imaging of a myc-expressing neutrophil population involved in inflammation and mycobacterial granuloma formation in zebrafish," Developmental and Comparative Immunology, vol. 32, no. 1, pp. 36-49, 2008.

[199] A. J. Sadler, O. Latchoumanin, D. Hawkes, J. Mak, and B. R. G. Williams, "An antiviral response directed by PKR phosphorylation of the RNA helicase A," PLoS Pathogens, vol. 5, no. 2, Article ID e1000311, 2009.

[200] A. Takaoka, Z. Wang, M. K. Choi et al., "DAI (DLM-1/ZBP1) is a cytosolic DNA sensor and an activator of innate immune response," Nature, vol. 448, no. 7152, pp. 501-505, 2007.

[201] V. Hornung, A. Ablasser, M. Charrel-Dennis et al., "AIM2 recognizes cytosolic dsDNA and forms a caspase-1-activating inflammasome with ASC," Nature, vol. 458, no. 7237, pp. 514-518, 2009. 
[202] V. Deretic, "Autophagy as an innate immunity paradigm: expanding the scope and repertoire of pattern recognition receptors," Current Opinion in Immunology, vol. 24, no. 1, pp. 21-31, 2012.

[203] D. M. Tobin, R. C. May, and R. T. Wheeler, "Zebrafish: a see-through host and a fluorescent toolbox to probe hostpathogen interaction," PLoS Pathogens, vol. 8, no. 1, Article ID e1002349, 2012.

[204] S. A. Renshaw and N. S. Trede, "A model 450 million years in the making: zebrafish and vertebrate immunity," Disease Models and Mechanisms, vol. 5, no. 1, pp. 38-47, 2012.

[205] K. J. Clark, D. F. Voytas, and S. C. Ekker, "A Tale of two nucleases: gene targeting for the masses?" Zebrafish, vol. 8, no. 3, pp. 147-149, 2011. 


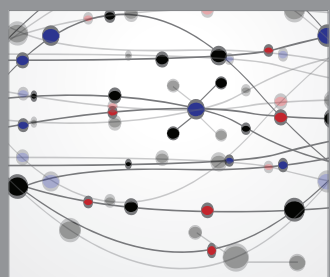

The Scientific World Journal
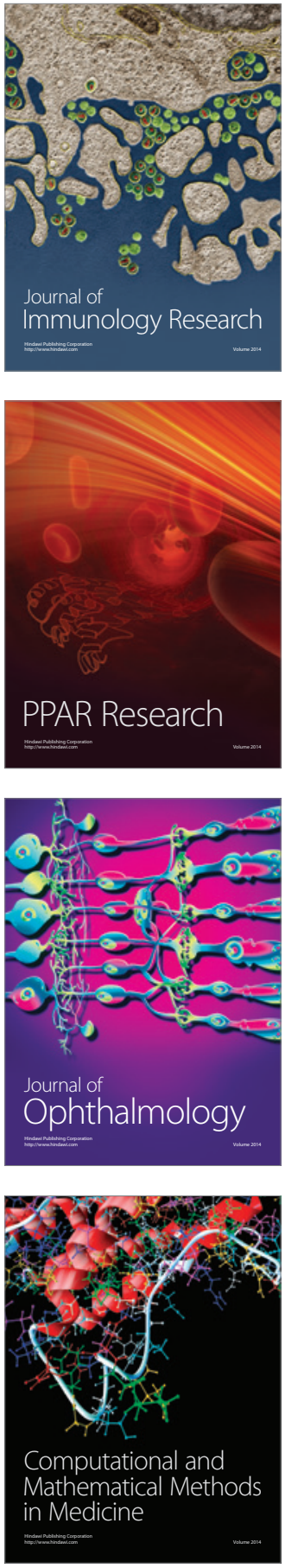

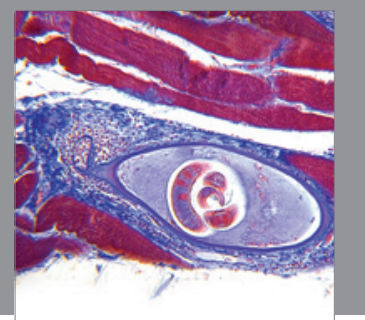

Gastroenterology

Research and Practice
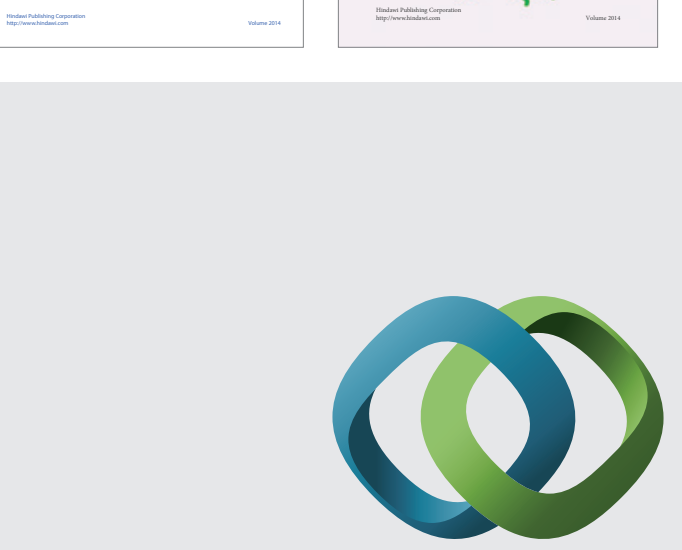

\section{Hindawi}

Submit your manuscripts at

http://www.hindawi.com
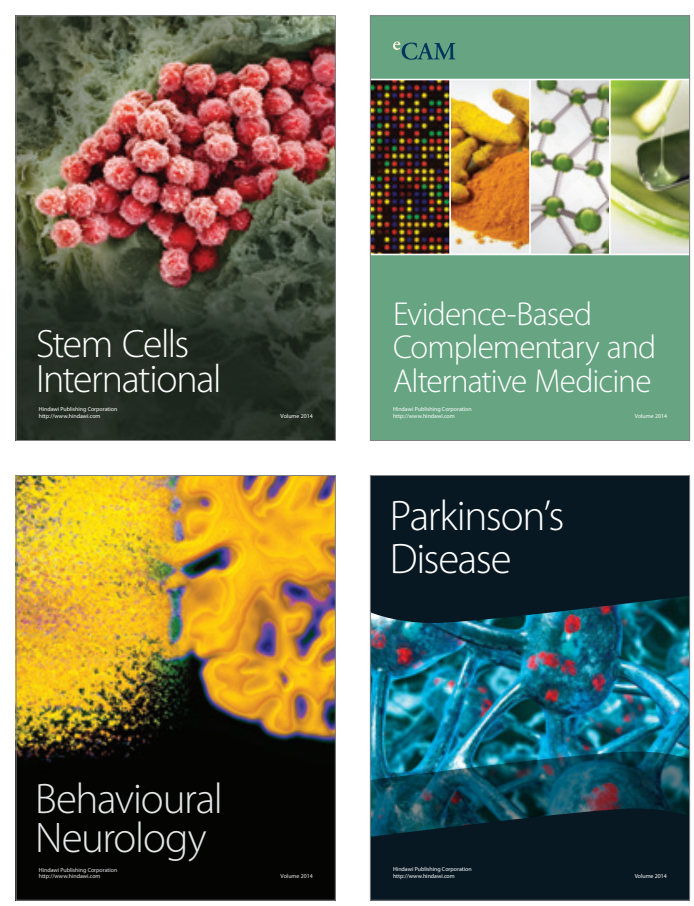

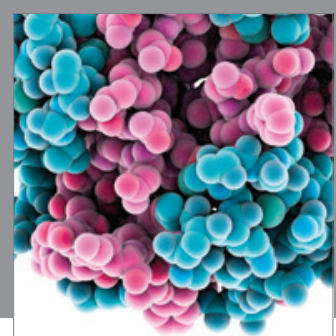

Journal of
Diabetes Research

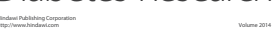

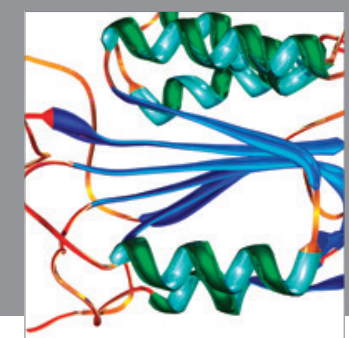

Disease Markers
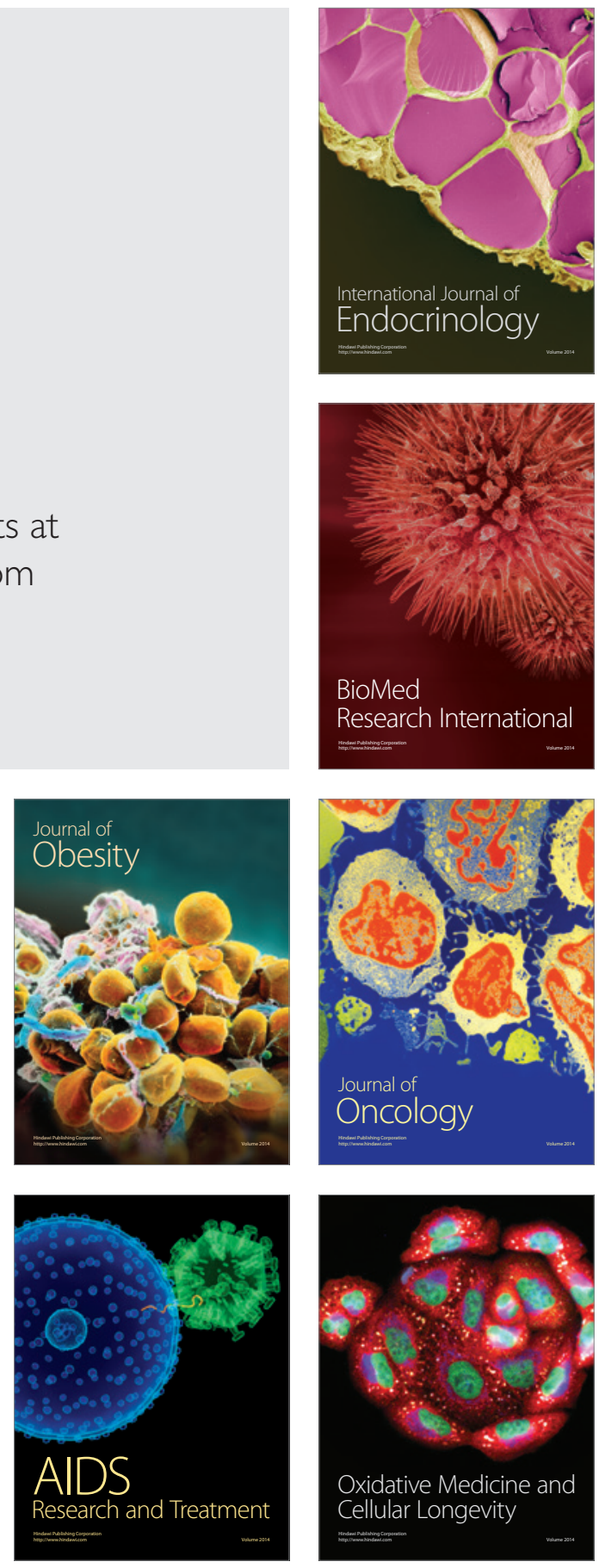